\title{
SPREADING WIDTHS OF ISOBARIC ANALOG STATES AND THE ISOVECTOR GIANT MONOPOLE RESONANCE
}

\author{
J. JÄNECKE \\ Department of Physics, University of Michigan, Ann Arbor, Michigan 48109, USA \\ M.N. HARAKEH \\ Kernfysisch Versneller Instituut, 9747 AA Groningen, The Netherlands \\ and
}

Natuurkundig Laboratorium, Vrije Universiteit, Postbus 7161, 1007 MC Amsterdam, The Netherlands

\section{S.Y. VAN DER WERF}

Kernfysisch Versneller Instituut, 9747 AA Groningen, The Netherlands

Received 23 July 1986

\begin{abstract}
A global analysis of the experimental spreading widths $\Gamma^{\downarrow}$ of 65 isobaric analog states (IAS) in the range $A=110$ to 238 has been performed assuming isospin mixing via the Coulomb force with $T_{<}=T_{0}-1$ doorway states mediated by coupling to the $\left(T_{0}-1\right)$-component of the giant isovector monopole resonance (IVM). Excellent agreement has been achieved over the entire range, thus establishing general smooth trends for the parameters describing the resonance. The excitation energy of the resonance, $E($ IVM $) \approx V_{0} / A^{1 / 3}-\left(T_{0}+1\right) V_{1} / A$ was found to reproduce the IAS data with $V_{0}$ in the range 155 to $170 \mathrm{MeV}$ and $V_{1} \approx 55 \mathrm{MeV}$. These values are taken from recent theoretical estimates of Auerbach and the extreme hydrodynamical estimate of Bohr, Damgaard and Mottelson. The charge-dependent matrix elements were found factors of two to three stronger than hydrodynamic or microscopic estimates, but in almost perfect agreement with an expression based on sum rules derived by Lane and Mekjian. A strength-function approach developed by MacDonald and Birse for the damping widths of isovector monopole resonances was successfully employed. However, it became necessary to introduce an explicit dependence on nuclear deformation to describe a splitting of the IVM strength due to coupling to the $\beta$-vibration component of the isovector quadrupole resonance (IVQ). The postulated $\beta$-dependence describes the strong increase with neutron excess of the spreading widths for several rare-earth nuclei, and an essentially one-parameter fit to all experimental spreading widths gives $\chi^{2} / f \approx 1.2$. It is further concluded that the density of doorway states which are responsible for the isospin mixing is much lower than the density of all underlying states of lower isospin with the same spin and parity as the IAS, and the ratio decreases exponentially with increasing excitation energy.
\end{abstract}

\section{Introduction}

The internal structure and other properties of isobaric analog states (IAS) have been discussed extensively in the literature [e.g. refs. ${ }^{1,2}$ )]. It was found, for example, that the total width $\Gamma$ ascribed to the lorentzian line shapes of IAS in medium-heavy and heavy nuclei can be expressed as a sum of two terms, the escape width $\Gamma^{\uparrow}$ and the spreading width $\Gamma^{\downarrow}$. The latter is of particular interest as it results from mixing via the charge-dependent Coulomb interaction with certain underlying states of lower isospin. Various models have been developed to describe this mixing ${ }^{3-10}$ ). 
The most prominent model assumes that isospin mixing is mediated by coupling to the $\left(T_{0}-1\right)$ component of the giant isovector monopole resonance (IVM) [refs. $\left.{ }^{6,7}\right)$ ]. Attempts to interpret the experimental data using this concept have been made [see e.g. refs. ${ }^{10-12}$ )]. Extensive data have become available in recent years ${ }^{11-18}$ ). This has prompted the present investigation which concentrates on a global interpretation of all experimental spreading widths for the heavier nuclei assuming isospin mixing with $T_{<}$doorway states mediated by coupling to the isovector monopole resonance (IVM).

The spreading widths of IAS are introduced in sect. 2. The data base is outlined in sect. 3. Implications of the so-called picket-fence model are discussed in sect. 4 . Isospin mixing introduced by the isovector monopole resonance (IVM) and the predicted properties of this resonance, particularly the strength function, are discussed in sect. 5. Also presented in this section are several expressions for the charge-dependent coupling matrix elements. The analysis of the spreading width data and the results are given in sect. 6 followed by a brief summary, sect. 7 .

\section{Spreading widths $\Gamma^{\downarrow}$ of isobaric analog states}

Isobaric analog states are simple modes of excitation of a nucleus. Their wave functions can be constructed by applying the isospin lowering operator $T_{-}$to the ground state of the parent nucleus. The coherent superposition of particle-hole excitations in an IAS preserves the simplicity of the parent nucleus ground state wave function. This is true despite the fact that in heavy nuclei IAS occur at high excitation energies of $\sim 15 \mathrm{MeV}$ and higher on account of the increased Coulomb displacement energies. However, small admixtures of contributions with lower isospin are usually present, and the wave functions of IAS are then written as ${ }^{3-11}$ ).

$$
|\mathrm{IAS}\rangle=\alpha_{T_{0}}\left|T_{0}\right\rangle+\alpha_{T_{0}-1}\left|T_{0}-1\right\rangle .
$$

Here, $T_{0}\left(=\frac{1}{2}|(N-Z)|\right)$ is the isospin of the parent nucleus (= the target nucleus in a $\left({ }^{3} \mathrm{He}, \mathrm{t}\right)$ charge-exchange reaction). The coefficient $\alpha_{T_{0}-1}$ of the second term is generally small with a square on the order of a few percent. It is the result of Coulomb and other charge-dependent interactions which mix the IAS with certain states with $T_{<}=T_{0}-1$. The coefficient $\alpha_{T_{0}-1}$ would disappear in the absence of these forces. The total width $\Gamma$ of an IAS can be written accordingly,

$$
\Gamma=\Gamma^{\hat{\imath}}+\Gamma^{\downarrow} \text {. }
$$

The escape width $\Gamma^{\uparrow}$ is the sum of all partial decay widths of the components with $T_{>}=T_{0}$ in the IAS. The spreading width $\Gamma^{\downarrow}$ results from mixing via the chargedependent Coulomb forces between states with $T_{<}=T_{0}-1$ and the IAS. It is usually not small and often bigger than the escape width. Neglecting $\gamma$ - and $\alpha$-decay, both widths may contain contributions from $T$-forbidden $\left(\Delta T=\frac{3}{2}\right)$ decays in addition to $T$-allowed $\left(\Delta T=\frac{1}{2}\right)$ nucleon decays. The $T$-forbidden decays are due to isospin mixing in the low-lying final states of the residual nuclei. The decay modes represented by $\Gamma^{\uparrow}$ are generally dominated by $T$-allowed proton decay into low-lying neutron 
hole states. Proton separation energies, the height of the Coulomb barrier, and the permitted angular momenta of the decay protons strongly affect the escape widths. Neutron decay is generally the dominating decay mode contributing to the spreading widths $\Gamma^{\downarrow}$. There are two contributions to neutron decay which cannot be distinguished. These are $T$-allowed decays from the $T_{<}$part of the IAS wave function and $T$-forbidden decays from the $T_{>}$part. Both of these decay modes have a similar origin, namely isospin mixing in either the initial IAS or in the final states of the residual nucleus. Their contributions will therefore be combined in $\Gamma^{\downarrow}$ in all further discussions. In heavier nuclei, fission also contributes to $\Gamma^{\downarrow}$ with typically $25 \%$ [ref. $\left.{ }^{11}\right)$ ]. Other types of decay which contribute to $\Gamma^{\uparrow}$ and $\Gamma^{\downarrow}$ such as $\gamma$-emission, $\alpha$-emission and $T$-forbidden emission of protons can usually be neglected on account of small matrix elements, the Coulomb barrier and binding energy systematics.

\section{Data base}

The most direct way to populate IAS in the heavier nuclei is through chargeexchange reactions such as $(\mathbf{p}, \mathbf{n})\left[\right.$ refs. $\left.\left.\left.{ }^{19,20}\right)\right],\left({ }^{3} \mathrm{He}, \mathrm{t}\right)\left[\mathrm{refs} .{ }^{21,11,13,14,17}\right)\right]$, or $\left(\pi^{+}, \pi^{0}\right)$ [refs. $\left.{ }^{22-25}\right)$ ] reactions. The angular momentum transfer is $L=0$, and the angular distributions are strongly forward peaked. Mainly even- $\boldsymbol{A}$ nuclei are reached in these reactions. Excitation functions for resonance reactions ${ }^{15,16,18,26-35}$ ) such as elastic or inelastic proton scattering via the IAS as well as one-nucleon pickup ${ }^{12}$ ) or stripping ${ }^{36}$ ) have also been used to study IAS. Mainly odd- $A$ nuclei are reached in these reactions.

Total widths $\Gamma$ can be extracted from the measured lorentzian line shapes or from proton elastic scattering resonance parameters. The dominant decay mode from the $T_{<}$component is isospin-allowed neutron emission, favored because of its large penetrability. Neutron emission can therefore serve as a direct measure of the spreading width $\Gamma^{\downarrow}$ of IAS. Fission is known to compete with neutron decay in the actinide nuclei ${ }^{11}$ ). Measuring the escape width $\Gamma^{\uparrow}$ via proton decay or deducing it from resonance parameters leads to indirect information about $\Gamma^{\downarrow}=\Gamma-\Gamma^{\uparrow}$. Estimates for $\Gamma^{\downarrow}$ can also be obtained from measured total widths $\Gamma$ and calculated ratios $\Gamma^{\downarrow} / \Gamma$ or $\Gamma^{\uparrow} / \Gamma$.

The majority of experimentally determined spreading widths $\Gamma^{\downarrow}$ of heavy nuclei and of essentially all even- $\boldsymbol{A}$ nuclei were derived from $\left({ }^{3} \mathrm{He}, \mathrm{t}\right)$ charge-exchange experiments. A few, however, were obtained by other techniques. The experimental data used in the present work are given in table 2 of the appendix.

Transitions to ground and excited IAS of seven odd-A In isotopes have been measured recently ${ }^{12}$ ) using the ${ }^{A} \mathrm{Sn}(\mathrm{p}, \mathrm{d}){ }^{A-1} \mathrm{Sn}$ neutron pickup reaction for all even- $A$ Sn targets from $A=112$ to 124 . The observed widths of the single-hole states were interpreted as essentially spreading widths $\Gamma^{\downarrow}$, but later work by Tohyama ${ }^{12}$ ) suggests that contributions from the escape width $\Gamma^{\uparrow}$ are not negligible and seem to account for the observed increase with neutron excess. 
Proton resonance experiments ${ }^{16,26,30,33,34}$ ) are the main source of information for the IAS of the odd- $A$ Sn and Te nuclei. Total and escape widths, $\Gamma$ and $\Gamma^{\dagger}$, are directly deduced from this work. Direct determinations of $\Gamma_{\mathrm{p}} \approx \Gamma^{\uparrow}$ in this mass region were also obtained from ( $\mathrm{p}, \mathrm{n} \overline{\mathrm{p}}$ ) experiments ${ }^{19,20}$ ). The widths $\Gamma_{\mathrm{p}}$ were found to be very small for the even- $A$ isotopes, $\leqslant 5 \mathrm{keV}$, but slightly increased for odd- $A$ isotopes, 6 to $24 \mathrm{keV}$. This behavior was ascribed ${ }^{21}$ ) to the systematics of the proton binding energies and hence decay probabilities. Combining this information with the extensive $\left({ }^{3} \mathrm{He}, \mathrm{t}\right)$ charge-exchange reaction data measured at $\theta=0^{\circ}$ for all even- $A$ and some odd- $A \mathrm{Sn}$ and Te isotopes ${ }^{21}$ ) lead to the conclusion that $\Gamma^{\uparrow} \ll \Gamma^{\downarrow}$ for the IAS of even- $A$ isotopes. The total widths $\Gamma$ of 10 to $40 \mathrm{keV}$ measured in the latter reaction were therefore interpreted as mostly spreading widths, $\Gamma^{\downarrow} \approx \Gamma$.

Spreading width $\Gamma^{\downarrow}$ of IAS in even- $A$ Pm isotopes were recently determined ${ }^{17}$ ) by measuring the ${ }^{A} \mathrm{Nd}\left({ }^{3} \mathrm{He}, \mathrm{t}\right){ }^{A} \mathrm{Pm}$ and ${ }^{A} \mathrm{Nd}\left({ }^{3} \mathrm{He}, \mathrm{t} \overline{\mathrm{p}}\right){ }^{A-1} \mathrm{Nd}$ charge-exchange reactions at $\theta=0^{\circ}$ for all even- $A$ Nd isotopes, $A=142$ to 150 . The total widths $\Gamma$ were determined by fitting the observed peaks by lorentzian line shapes and correcting these for the instrumental width as determined from the peak widths of light contaminants, ${ }^{12} \mathrm{C}$ and ${ }^{16} \mathrm{O}$. Comparing the double differential cross section for proton decay $\left({ }^{3} \mathrm{He}, \mathrm{t} \overline{\mathrm{p}}\right)$ to that for $\left({ }^{3} \mathrm{He}, \mathrm{t}\right)$ makes it possible to determine the IAS branching ratios or proton decay probabilities $P^{\uparrow}$. The escape and spreading widths are then obtained from $\Gamma^{\uparrow}=P^{\uparrow} \Gamma$ and $\Gamma^{\downarrow}=\Gamma-\Gamma^{\uparrow}$. The total widths $\Gamma$ of the IAS were found to range from 33 to $100 \mathrm{keV}$, the spreading widths $\Gamma^{\downarrow}$ from 28 to $65 \mathrm{keV}$. Interestingly, in the most neutron-rich isotope the escape width contributes about $30 \%$ to the total width.

The total widths $\Gamma$ of IAS in 24 even- $A$ rare-earth nuclei from ${ }^{144} \mathrm{Sm}$ to ${ }^{180} \mathrm{Hf}$ were determined with the $\left({ }^{3} \mathrm{He}, \mathrm{t}\right)$ charge-exchange reaction $\left.{ }^{13,14}\right)$ at $\theta=0^{\circ}$ and were found in the range 30 to $110 \mathrm{keV}$. They increase with neutron excess within each sequence of isotopes. Contributions $\Gamma^{\uparrow} \approx \Gamma_{\mathrm{p}}$ can be estimated for the odd- $\boldsymbol{A}$ rareearth nuclei from the experimental partial proton decay widths $\Gamma_{\mathrm{p}}$ obtained from proton resonance experiments ${ }^{35}$ ). They are on the average about $4 \mathrm{keV}$ for $l=1$ $\left(\Gamma_{\mathrm{p}} / \Gamma \approx 0.04\right)$ and $2 \mathrm{keV}$ for $l=3\left(\Gamma_{\mathrm{p}} / \Gamma \approx 0.02\right)$, and the partial proton decay widths $\Gamma_{\mathrm{p}}$ for even- $A$ nuclei with their reduced proton decay energies should therefore not exceed a few keV justifying $\Gamma^{\downarrow} \approx \Gamma$ [refs. ${ }^{13,14}$ )]. However, the data obtained recently for the even- $A$ Nd isotopes ${ }^{17}$ ) suggest that this assumption may overestimate the spreading widths $\Gamma^{\downarrow}$ for the neutron-rich $\mathrm{Sm}$ to $\mathrm{Hf}$ isotopes by as much as $30 \%$. The reported values, marked with the $\leqslant$ sign in table 2 , will nevertheless be used in the present work as its major purpose is to deduce general trends of the various parameters which describe the isospin mixing with the isovector monopole resonance.

Additional experimental spreading widths $\Gamma^{\downarrow}$ for ${ }^{181} \mathrm{Ta},{ }^{208,209} \mathrm{~Pb},{ }^{232} \mathrm{Th}$ and ${ }^{238} \mathrm{U}$ are used in the present work. They range from $70 \mathrm{keV}$ to $140 \mathrm{keV}$. The spreading widths for the IAS of ${ }^{181} \mathrm{Ta}$ and ${ }^{208} \mathrm{~Pb}$ were again measured $\left.{ }^{37,38}\right)$ with the $\left({ }^{3} \mathrm{He}, \mathrm{t}\right)$ and $\left({ }^{3} \mathrm{He}, \mathrm{t} \overline{\mathrm{p}}\right)$ charge-exchange reactions at $\theta=0^{\circ}$ on ${ }^{181} \mathrm{Ta}$ and ${ }^{208} \mathrm{~Pb}$ targets. The value $\Gamma^{\downarrow}=90 \pm 15 \mathrm{keV}\left[\right.$ ref. $\left.\left.{ }^{38}\right)\right]$ for the IAS of ${ }^{208} \mathrm{~Pb}$ is in good agreement with the 


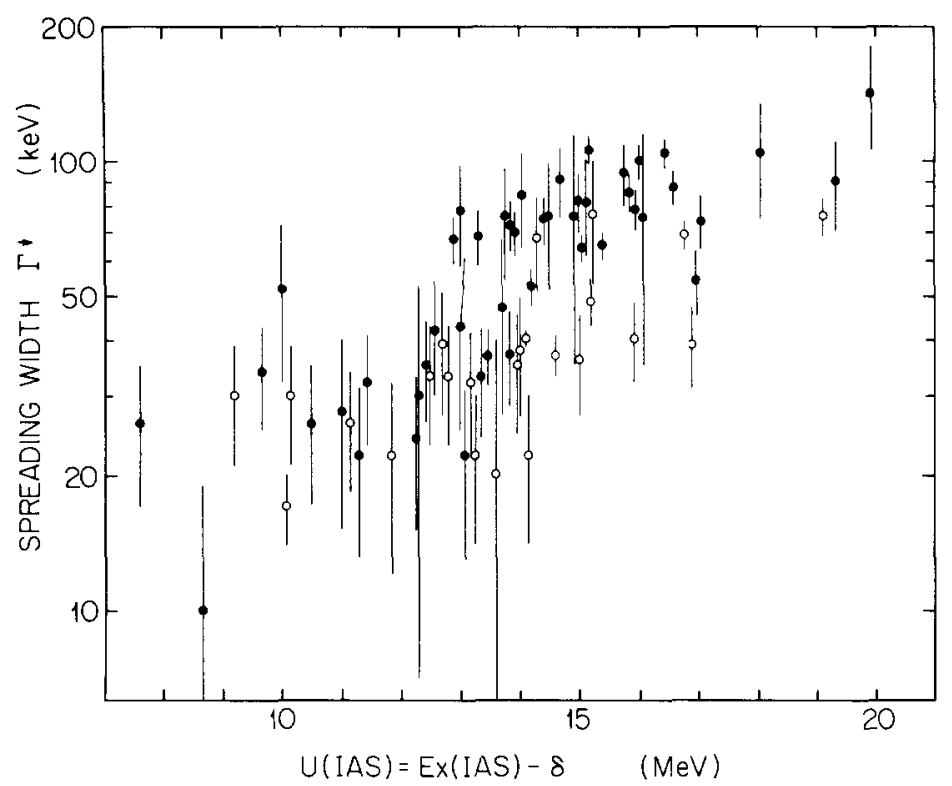

Fig. 1. Spreading widths $\Gamma^{\downarrow}$ of isobaric analog states for $A \approx 110$ to 238 as function of the "back-shifted" excitation energies $\mathrm{U}(\mathrm{IAS})=E_{\mathbf{x}}$ (IAS) $-\delta$. Values for the IAS of even-even and odd- $A$ nuclei are given by filled and open circles, respectively. Total widths $\Gamma$ are plotted for several nuclei (see text and table 2 ).

value $\Gamma^{\downarrow}=78 \pm 8 \mathrm{keV}$ cited by Melzer et al. ${ }^{18}$ ) as an average of elastic and inelastic proton resonant-scattering measurements. The latter value was used in the present global analysis. The spreading width for the IAS of ${ }^{209} \mathrm{~Pb}$ is from recent cross section and analyzing power excitation function measurements $\left.{ }^{18}\right)$ for ${ }^{208} \mathrm{~Pb}\left(\overrightarrow{\mathrm{p}}, \mathrm{p}_{0}\right)^{208} \mathrm{~Pb}$.

The spreading widths of the IAS of ${ }^{232} \mathrm{Th}$ and ${ }^{238} \mathrm{U}$ were obtained by comparing the measured $\left.{ }^{11}\right)$ double differential cross sections for fission decay, $\left({ }^{3} \mathrm{He}, \mathrm{tf}\right)$, to the cross sections for $\left({ }^{3} \mathrm{He}, \mathrm{t}\right)$. The fission decay probability from the $T_{<}$part of the IAS wave function includes contributions from 1st-, 2nd- and 3rd-chance fission. The results were compared [see refs. $\left.{ }^{11,39}\right)$ ] to statistical model calculations thus allowing the determination of the spreading widths of $\Gamma^{\downarrow}$ of the two IAS.

The data points are displayed in fig. 1 as a function of the pairing-energy-corrected excitation energies $U=E_{\mathrm{x}}-\delta$ (see below). The correction is used because the data include isobaric analog states of both even-even and odd- $\boldsymbol{A}$ nuclei. The widths of both types of nuclei increase from about 20 to $100 \mathrm{keV}$ for excitation energies from 10 to $20 \mathrm{MeV}$.

\section{Picket-fence model}

The spreading width of analog states may be described, at least in the absence of external mixing, as a "microgiant" resonance by using a picket-fence model ${ }^{3,5}$ ). 
Assuming that the states with $T_{<}=T_{0}-1$ have equal spacing $D$ and couple with the same off-diagonal charge-dependent matrix element $V_{\mathrm{CD}}=M_{\mathrm{AD}}$ to the IAS, the distribution of analog strength into these $T_{<}$states is a lorentzian of width ${ }^{5}$ )

$$
\Gamma^{\downarrow}=2 \pi \frac{\left\langle V_{\mathrm{CD}}^{2}\right\rangle}{D}\left(1+\frac{D^{2}}{\pi^{2}\left\langle V_{\mathrm{CD}}^{2}\right\rangle}\right)^{1 / 2}
$$

The energy spacing $D$ and level density $\rho=1 / D$ of all $T_{<}$states near $E_{\mathrm{x}}$ (IAS) can be estimated for any $J^{\pi}$ quite reliably using the "back-shifted Fermi-gas formula" ${ }^{40-42}$ ). The formula is believed to yield reasonable estimates up to excitation energies $U=E_{\mathrm{x}}-\delta$ of 15 to $20 \mathrm{MeV}$. It avoids the problem of the conventional Fermi-gas model of predicting level densities which are too high at excitation energies above $15 \mathrm{MeV}$. For the present calculations the pairing energies $\delta$ were obtained as suggested earlier ${ }^{42}$ ), and the level density parameters $a$ were obtained from a smooth interpolation of the experimentally determined values. The level densities up to 15 or $20 \mathrm{MeV}$ may still reflect upon the shell structure of the nucleus near its ground state.

The calculated level densities near the excitation energies $E_{\mathrm{x}}$ (IAS) range from about $0.3 / \mathrm{eV}$ at $U=10 \mathrm{MeV}$ to about $600 / \mathrm{eV}$ at $U=15 \mathrm{MeV}$. Hence, if one assumes a constant charge-dependent matrix element $V_{\mathrm{CD}}$, and furthermore that mixing takes place with all underlying $T_{<}$states of the same $J^{\pi}$, the width $\Gamma^{\downarrow}$ should increase by over three orders of magnitude. An increase by only a factor of three to four is observed. The same discrepancy prevails if it is assumed that a constant fraction $f$ of all states, the underlying (doorway) states, mix with the IAS via a constant charge-dependent interaction $V_{\mathrm{CD}}$.

In order to obtain agreement with the experimental $\Gamma^{\downarrow}$ one has to assume that $V_{\mathrm{CD}}$ and/or the ratio of doorway states (certain $1 \mathrm{p}-1 \mathrm{~h}$ and $2 \mathrm{p}-2 \mathrm{~h}$ states; see below) to all states with the same $J^{\pi}, f \equiv \rho_{\text {doorway }}\left(E_{\mathrm{IAS}} ; J^{\pi}\right) / \rho\left(E_{\mathrm{IAS}} ; J^{\pi}\right)$, decrease with increasing excitation energy and mass number. Assuming $f=$ const $\leqslant 1$ requires the matrix elements $V_{\mathrm{CD}}$ to decrease by many orders of magnitude. Such a strong decrease in $V_{\mathrm{CD}}$ has been introduced ${ }^{43,44}$ ) [see also ref. ${ }^{45}$ )] but appears unlikely considering that the internal structure of the IAS and of the doorway states which are mixing with the IAS remain essentially unchanged with mass number. It is interesting to note that the data for isospin-symmetry-breaking compound nucleus reactions seem to favor a parameterization in terms of a spreading width rather than a Coulomb matrix element ${ }^{45,46}$ ).

Assuming $V_{C D} \approx$ const, an assumption supported by the analysis below, the density of doorway states becomes directly proportional to the measured $\Gamma^{\downarrow}$. Values in the range 1 to $8 \mathrm{keV}$ are quoted in the literature for light nuclei, $A<40$ [see e.g. ref. ${ }^{45}$ )]. Similar values are also reported for isospin-forbidden beta-decays in light and heavy nuclei [see e.g. ref. ${ }^{47}$ )]. Thus, fig. 1 reflects directly upon the increase of the density of doorway states with excitation energy and mass number. Using for example $V_{\mathrm{CD}} \approx 2 \mathrm{keV}$, the fraction $f$ of doorway states needed to obtain agreement with the 
measured values $\Gamma^{\downarrow}$ has been determined for all even- $A$ and odd- $A$ data. The "back-shifted Fermi-gas formula" ${ }^{40-42}$ ) was used to calculate the density of all states. The result is shown in fig. 2 where $f$ is plotted as a function of the "backshifted" excitation energy $U($ IAS $)=E_{\mathrm{x}}($ IAS $)-\delta$. A strong correlation can clearly be seen. The fraction $f$ decreases by about one order of magnitude for every $1.7 \mathrm{MeV}$ in excitation energy and can approximately be represented by $f \approx \exp (-1.35(U-$ $7 \mathrm{MeV})$ ). The odd- $A$ nuclei follow essentially the same dependence as the even-even nuclei even though the IAS have both positive and negative parity and the spins $J$ range from $\frac{1}{2}$ to $\frac{9}{2}$. The values for ${ }^{208} \mathrm{~Pb}$ and ${ }^{209} \mathrm{~Pb}$ do not follow the general trend. They are about three orders of magnitude too high because the level density parameters $a$ of the back-shifted Fermi-gas formula derived from the level densities at low excitation energies are exceptionally small. The formula should be used with caution for $E_{\mathrm{x}}>15 \mathrm{MeV}$. Similarly, the values for ${ }^{232} \mathrm{Th}$ and ${ }^{238} \mathrm{U}$ appear about two orders of magnitude too low because the parameters $a$ are high. If the ratios $f$ for these four nuclei in fig. 2 are required to follow the general trend, the level density parameters have to be readjusted. The values $a \approx 19.9 / 18.9 / 21.6 / 22.4 \mathrm{MeV}^{-1}$ for ${ }^{208} \mathrm{~Pb} /{ }^{209} \mathrm{~Pb} /{ }^{232} \mathrm{Th} /{ }^{238} \mathrm{U}$ are much more in line with the general $A$-dependence of these parameters than the unadjusted values $a \approx 10.0 / 10.5 / 28.1 / 26.6 \mathrm{MeV}^{-1}$.

Plotting the ratio $f$ as function of $(A U)^{1 / 2}$ (not shown) displays a decrease similar to that of fig. 2 except that the even- $A$ and odd- $A$ data are slightly shifted relative to each other. The result $f \approx \exp \left(-0.42\left((A U)^{1 / 2}-26.5 \mathrm{MeV}^{1 / 2}\right)\right)$ is in approximate

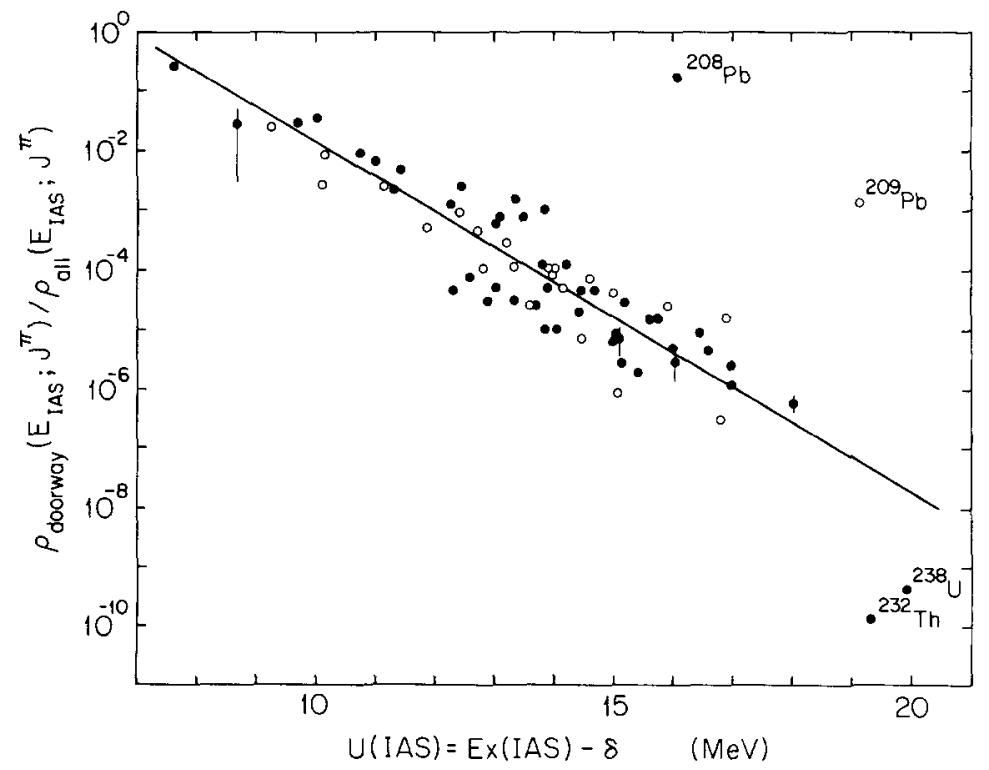

Fig. 2. Ratio of the density of doorway states for spreading to the density of all states of lower isospin but the same $J^{\pi}$ as the IAS as function of the "back-shifted" excitation energies $U($ IAS $)=E_{\mathbf{x}}($ IAS) $-\delta$. A charge-dependent matrix element $V_{C D} \approx 2 \mathrm{keV}$ is assumed (see text). 
numerical agreement with the function used by Kuhlmann ${ }^{44}$ ) to describe the decrease of $V_{\mathrm{CD}}$.

The density of doorway states is believed to have a maximum at higher excitation energies (see below). The densities at $E_{\mathrm{x}}$ (IAS) in the low-energy tail region of this distribution increases only slowly with mass number at a rate which is much slower than the density of all states with lower isospin and the same $J^{\pi}$ as the IAS. It is therefore not surprising to find that the ratio $f$ becomes increasingly much less than unity and is approximately inversely proportional to the total level density at $E_{\mathrm{x}}$ (IAS).

\section{Isospin mixing with the giant isovector monopole resonance}

\subsection{GENERAL CONSIDERATIONS}

It is generally accepted ${ }^{6,7}$ ), that the spreading width $\Gamma^{\downarrow}$ of IAS is due to mixing via the Coulomb and possibly other charge-dependent interactions between the IAS with isospin $T=T_{0}$ and states with $T_{<}=T_{0}-1$. Two types of $T_{<}$states are involved. The so-called configuration or anti-analog states have an intrinsic particle-hole structure similar to but orthogonal to that of the IAS and seem to dominate the mixing in light nuclei $\left.{ }^{6}\right)$. The $\left(T_{0}-1\right)$-component of the postulated $\left.{ }^{6.7}\right)$ and recently observed $^{22-25}$ ) giant charge-exchange isovector monopole resonance (IVM) is believed to dominate the mixing in heavy nuclei because of its close relation to the intrinsic nuclear structure of the IAS. In a microscopic description this resonance involves a coherent linear superposition of $(J=0)$-coupled neutron-hole/protonparticle states with different radial quantum numbers, $\Delta n_{\mathrm{r}}=1$ (all other quantum numbers for the hole/particle pairs are the same). This is a $2 \hbar \omega$ excitation with no change in $J^{\pi}$. In a hydrodynamic description the isovector monopole resonance can be viewed as a breathing mode without change in shape where neutrons and protons oscillate out of phase. However, this picture is complicated by the fact that the charge-exchange monopole resonance is split into three components with isospins of $T_{0}+1, T_{0}$, and $T_{0}-1$ due to the coupling of the $\tau=1$ isovector monopole excitation with the isospin $T_{0}$ of the core. The splitting is usually described as symmetry energy splitting. Based on the parent nucleus with $T_{z}=T_{0}$, all three components of the IVM are present in the proton-rich neighboring isobar with $T_{z}=T_{0}-1$, whereas only two and one components are present in the isobars with $T_{z}=T_{0}$ and $T_{z}=T_{0}+1$, respectively (see fig. 3 ). Corresponding components of the IVM are shifted relative to each other due to a Coulomb energy displacement similar to the shift between IAS and target GS.

Neglecting contributions from configuration states, the spreading widths of the IAS in heavier nuclei are approximately described by ${ }^{6,7}$ ),

$$
\Gamma^{\downarrow} \approx V_{\mathrm{CD}}^{2} \frac{\Gamma_{\mathrm{IVM}}}{(\Delta E)^{2}+\left(\frac{1}{2} \Gamma_{\mathrm{IVM}}\right)^{2}} .
$$




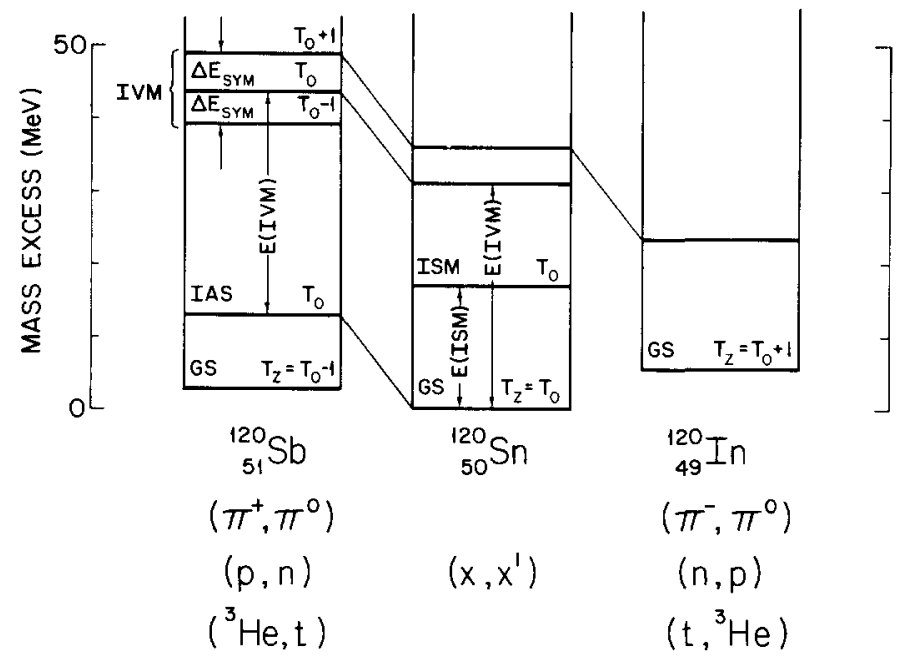

Fig. 3. Schematic level scheme of ground and isobaric analog states (experimental), of the isoscalar monopole resonance, and of the three components of the isovector monopole resonance for ${ }^{120} \mathrm{Sn}$ and its two neighboring isobars. The latter are calculated from eq. (5) with $V_{0}=155 \mathrm{MeV}$ and $V_{1}=55 \mathrm{MeV}$ (see also fig. 4).

Here, $V_{\mathrm{CD}}=M_{\mathrm{AM}}=\left\langle\mathrm{IVM}_{T_{0}-1}\left|H_{\mathrm{AM}}\right| \mathrm{IAS}\right\rangle$ is the charge-dependent matrix element which mixes the $\left(T_{0}-1\right)$ component of the IVM resonance with the IAS. The quantity $\Delta E$ represents the energy difference between the excitation energies of the $\left(T_{0}-1\right)$ component of the IVM and the IAS, $\Delta E=E_{\mathrm{x}}\left(\mathrm{IVM}_{T_{0}-1}\right)-E_{\mathrm{x}}(\mathrm{IAS})$. The energydependent damping width $\Gamma_{\mathrm{IVM}}$ of the $\left(T_{0}-1\right)$ component of the IVM has to be obtained at $E_{\mathrm{x}}=E_{\mathrm{x}}$ (IAS). It has the characteristics of a strength function.

The energy differences between the three components of the IVM with $T=T_{0}-1$, $T_{0}$, and $T_{0}+1$ and the IAS are usually parameterized as ${ }^{48,7,9}$ )

$$
E_{\mathrm{IVM}}(T)-E_{\mathrm{IAS}}\left(T_{0}\right)=V_{0} A^{-1 / 3}+\frac{1}{2}\left[T(T+1)-T_{0}\left(T_{0}+1\right)-2\right] V_{1} A^{-1}
$$

and in particular

$$
E_{\mathrm{IVM}}\left(T_{0}-1\right)-E_{\mathrm{IAS}}\left(T_{0}\right)=V_{0} A^{-1 / 3}-\left(T_{0}+1\right) V_{1} A^{-1} .
$$

Here, the first term is the centroid energy for the three components of the IVM,

$$
\overline{E_{\mathrm{IVM}}(T)}-E_{\mathrm{IAS}}\left(T_{0}\right)=V_{0} A^{-1 / 3} \text {. }
$$

It decreases weakly with increasing mass number. The simple shell-model estimate for $V_{0}$ (excitations of $2 \hbar \omega$ ) is $V_{0} \approx 82 \mathrm{MeV}$, but the hydrodynamic model which yields $V_{0} \approx 170 \mathrm{MeV}^{49}$ ) is considered more realistic as it reflects upon the expected strong collectivity. The second term in eqs. (5) and (6) is primarily due to symmetry energy splitting. The coefficient $V_{1}$ obtained from a single-particle symmetry potential ${ }^{48}$ ) is about $V_{1} \approx 100 \mathrm{MeV}$. This value corresponds to that used in mass equations. Collective effects reduce the splitting ${ }^{50}$ ), and other estimates ${ }^{50,51}$ ) are $V_{1} \approx 55 \mathrm{MeV}$ 
and $V_{1}=60 \pm 9 \mathrm{MeV}$. Recent microscopic calculations based on the random phase approximation (RPA) [ ref. $^{52}$ )] have been used to estimate the energies and widths of the components of IVM for ${ }^{48} \mathrm{Ca},{ }^{90} \mathrm{Zr},{ }^{120} \mathrm{Sn}$ and ${ }^{208} \mathrm{~Pb}$. These values are approximately compatible with $V_{0} \approx 155 \mathrm{MeV}$ and $V_{1} \approx 55 \mathrm{MeV}$.

While postulated about 15 years ago ${ }^{5,7}$ ), experimental confirmation of certain properties of the IVM have become available only very recently ${ }^{22-25}$ ). Using the $\left(\pi^{-}, \pi^{0}\right)$ charge-exchange reaction on targets of ${ }^{60} \mathrm{Ni},{ }^{90} \mathrm{Zr},{ }^{120} \mathrm{Sn},{ }^{140} \mathrm{Ce}$, and ${ }^{208} \mathrm{~Pb}$, observation of the $\left(T_{0}+1\right)$ component of the IVM [refs. $\left.\left.{ }^{22-25}\right)\right]$ in the neighboring isobars with $T_{\mathrm{z}}=T_{0}+1$ has been reported with excitation energies in reasonable agreement with predicted energies. More limited information from the $\left(\pi^{+}, \pi^{0}\right)$ reaction has also been reported $\left.{ }^{24,25}\right)$ for the $\left(T_{0}-1\right)$ components in the neighboring isobars with $T_{z}=T_{0}-1$. Fig. 4 displays the dependence on $A$ of the excitation energies $E_{\mathrm{IVM}}(T)-E_{\mathrm{IAS}}\left(T_{0}\right)$ from eq. (5) with $V_{0}=155 \mathrm{MeV}$ and $170 \mathrm{MeV}$ and $V_{1}=55 \mathrm{MeV}$. Results from RPA calculations ${ }^{52}$ ) are included for four select nuclei. Also shown are the experimental excitation energies from the $\left(\pi^{-}, \pi^{0}\right)$ chargeexchange reaction ${ }^{25}$ ) corrected for the differences between IVM centroid energies and cross section maxima (from fig. 14 of ref. ${ }^{25}$ ).

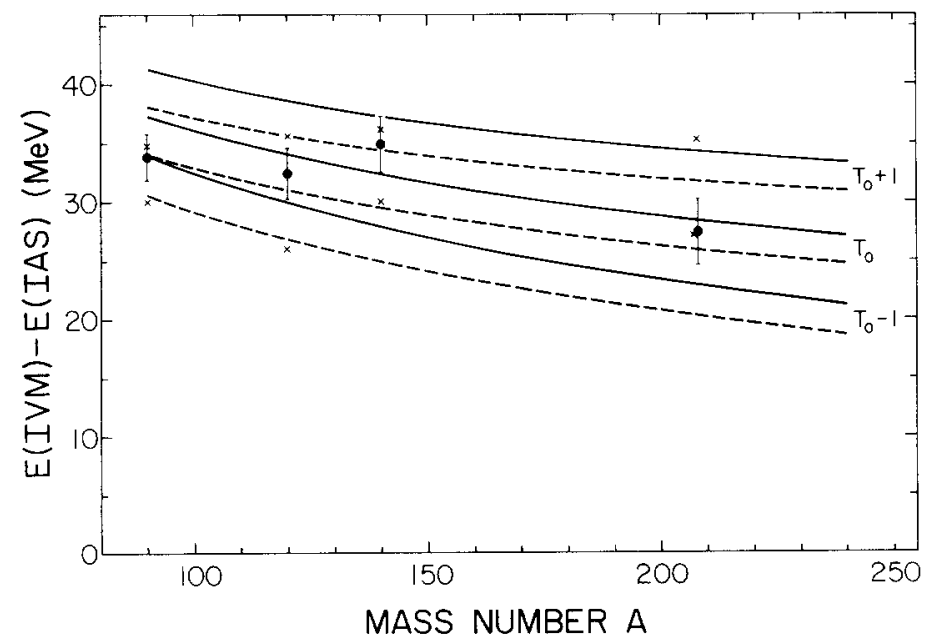

Fig. 4. Calculated energies $E_{\mathrm{IVM}}(T)-E_{\mathrm{IAS}}\left(T_{0}\right)$ from eq. (5) for $T=T_{0}+1, T_{0}$ and $T_{0}-1$ with $V_{0}=$ $170 \mathrm{MeV}$ (solid lines) and $V_{0}=155 \mathrm{MeV}$ (dashed lines) and $V_{1}=55 \mathrm{MeV}$. Also included are values (x) for $T=T_{0} \pm 1$ from RPA calculations ${ }^{52}$ ) for four select nuclei and experimental values for $T=T_{0}+1$ derived from $\left(\pi^{-}, \pi^{0}\right)$ charge-exchange reactions $\left.{ }^{25}\right)$.

Estimates of the damping widths $\Gamma_{\text {IVM }}$ are based on a variety of considerations ${ }^{52-56,2,10}$ ) including microscopic and macroscopic models, HartreeFock calculations, the random phase approximation, sum rule approaches, and hydrodynamic models. Values in the range $6 \mathrm{MeV}$ to $15 \mathrm{MeV}$ seem to be typical, whereas values of $2 \mathrm{MeV}$ to $3 \mathrm{MeV}$ are needed ${ }^{6}$ ) to account for the experimentally 
observed spreading widths $\Gamma^{\downarrow}$ of the IAS. This discrepancy has persisted for many years. Recent work by Auerbach and Klein ${ }^{52}$ ) and by MacDonald and Birse ${ }^{10}$ ) deals explicitly with the energy dependence of $\Gamma_{\mathrm{IVM}}$ and the distribution of strength using microscopic theories and sum rule approaches. The latter formulation has been used in the present work because it permits a simple parameterization of the dependence on charge and mass.

\subsection{THE STRENGTH FUNCTION}

Eq. (4) for the spreading width $\Gamma^{\downarrow}$ of IAS of heavier nuclei can be rewritten as

$$
\Gamma^{\downarrow}=2 \pi V_{\mathrm{CD}}^{2} S\left(E_{\mathrm{IAS}}\right) \text {. }
$$

Here, $V_{\mathrm{CD}}=M_{\mathrm{AM}}$ is the matrix element which couples the IAS with the IVM, and

$$
S(E)=\langle\operatorname{IVM}| \text { doorway }\rangle_{E}^{2} \rho_{\text {doorway }}(E)
$$

is the energy-dependent strength function which describes the distribution of the IVM wave function over the bound and continuum states of the nucleus. These doorway states (spreading state) which mix with the IVM are certain $1 \mathrm{p}-1 \mathrm{~h}$ and $2 \mathrm{p}-2 \mathrm{~h}$ states. In the strength function approach based on sum rules $\left.{ }^{10}\right), S(E)$ is given by

$$
S(E)=\frac{1}{2 \pi} \frac{\Gamma_{\mathrm{IVM}}(E)}{\left(E-E_{\mathrm{IVM}}-\Delta_{\mathrm{IVM}}(E)\right)^{2}+\left(\frac{1}{2} \Gamma_{\mathrm{IVM}}(E)\right)^{2}},
$$

where $\Gamma_{\mathrm{IVM}}(E)$ and $\Delta_{\mathrm{IVM}}(E)$ are respectively the width and shift functions. In particular,

$$
\Gamma_{\mathrm{IVM}}(E)=2 \pi\left\langle\mathrm{IVM}\left|H_{M D}\right| \text { door }\right\rangle_{E}^{2} \rho_{\text {door }}(E)
$$

represents the distribution of coupling strength between the IVM and the doorway states (spreading state). The matrix element $M_{\mathrm{MD}}=\left\langle\mathrm{IVM}\left|H_{\mathrm{MD}}\right|\right.$ door $\rangle$ is due to the residual nuclear interaction which strongly couples the collective $1 \mathrm{p}-1 \mathrm{~h}$ IVM state to $2 \mathrm{p}-2 \mathrm{~h}$ configurations. The above width is not constant as it would violate a sum rule ${ }^{10}$ ). Instead, it can be approximated by

$$
\Gamma_{\mathrm{IVM}}(E) \approx M_{2} \frac{\Gamma_{\mathrm{s}}}{\left(E-E_{\mathrm{s}}\right)^{2}+\left(\frac{1}{2} \Gamma_{\mathrm{s}}\right)^{2}}
$$

and similarly for the shift function,

$$
\Delta_{\mathrm{IVM}}(E) \approx M_{2} \frac{\left(E-E_{\mathrm{s}}\right)}{\left(E-E_{\mathrm{s}}\right)^{2}+\left(\frac{1}{2} \Gamma_{\mathrm{s}}\right)^{2}} .
$$

Here,

$$
M_{2}=\int\left(E-E_{\mathrm{IVM}}\right)^{2} S(E) \mathrm{d} E
$$

is the second moment of the strength function. The strength function $S(E)$ of eq. (10) has a quasi-Breit-Wigner shape which falls off in the tail as $\left(E-E_{\mathrm{IVM}}\right)^{-4}$. A 
Breit-Wigner shape with $\Gamma_{\mathrm{IVM}}=$ const. would lead to $M_{2} \rightarrow \infty$, and a gaussian shape would give $\Gamma_{\mathrm{IVM}} \propto\left(M_{2}\right)^{1 / 2}$. Using the results from RPA calculations for four spherical nuclei from ${ }^{16} \mathrm{O}$ to ${ }^{208} \mathrm{~Pb}^{57,58}$ ), simple approximate parameterizations can be introduced ${ }^{10}$ ),

$$
\begin{aligned}
M_{2} & \approx 547.9 A^{-0.4558} \mathrm{MeV}^{2}, \\
\bar{E}_{\mathrm{s}} & \approx \bar{E}_{\mathrm{IVM}}+2.5 \mathrm{MeV}, \\
\frac{1}{2} \Gamma_{\mathrm{s}} & \approx 2 \hbar \omega=82 A^{-1 / 3} \mathrm{MeV} .
\end{aligned}
$$

Eqs. (11) to (17) show that the centroid of the coupling strength, $\bar{E}_{\mathrm{s}}$, is located slightly above $\bar{E}_{\mathrm{IVM}}$, that the matrix elements from pure shell model states extend to configurations approximately $2 \hbar \omega$ away, but most importantly, that $\Gamma_{\mathrm{IVM}}\left(E_{\mathrm{IAS}}\right)$ is significantly smaller than $\Gamma_{\mathrm{IVM}}\left(E_{\mathrm{IVM}}\right)$. Fig. 5 displays for $A=120$ the calculated distribution of coupling strength $\Gamma_{\mathrm{IVM}}(E)$ between the $\left(T_{0}-1\right)$ component of the IVM and the doorway states (spreading state) as well as the distribution $S(E)$ of the IVM wave function over the bound and unbound states.

Eq. (8) with eq. (10) and the parameterizations of eqs. (12) to (17) will be used in the analyses presented below.

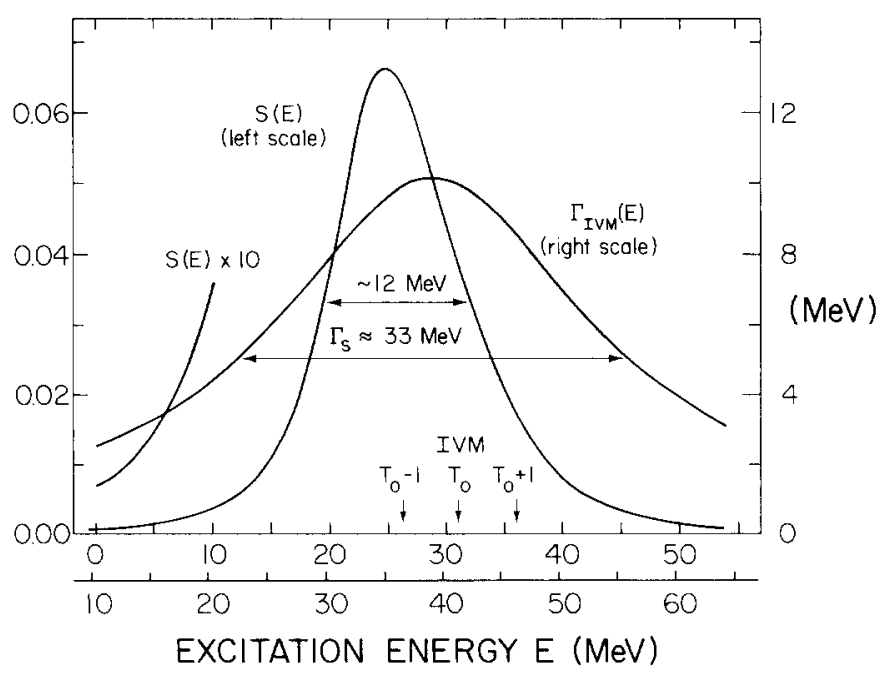

Fig. 5. Calculated dependence of the width function $\Gamma_{1 \vee M}(E)$ and the strength function $S(E)$ on the excitation energy relative to the IAS for $A=120$. The width function, assumed to have a lorentzian line shape, represents the distribution of IVM coupling strength into the doorway states. The strength function describes the spread of the IVM wave function into the doorway states. It has a quasi-Breit-Wigner line shape.

\subsection{THE CHARGE-DEPENDENT MATRIX ELEMENT}

The Coulomb matrix element $V_{\mathrm{CD}}=M_{\mathrm{AM}}$ in eqs. (4) and (8) describes the coupling between the IVM and the IAS. It can be written as a product of a vector coupling 
coefficient and a reduced matrix element,

$$
V_{\mathrm{CD}}^{2} \equiv M_{\mathrm{AM}}^{2}=C^{2}\left(T_{0} T_{0}-110 \mid T_{0}-1 T_{0}-1\right) \mathcal{M}
$$

Here

$$
\begin{gathered}
M_{\mathrm{AM}}^{2}=\left\langle\operatorname{IVM}\left(T_{0}-1\right)\left|H_{\mathrm{CD}}\right| \operatorname{IAS}\left(T_{0}\right)\right\rangle^{2}, \\
C^{2}\left(T_{0} T_{0}-110 \mid T_{0}-1 T_{0}-1\right)=\frac{2 T_{0}-1}{T_{0}\left(2 T_{0}+1\right)}, \\
\mathcal{M}=\left\langle\operatorname{IVM}\left(T_{0}-1\right)\left\|H_{\mathrm{CD}}\right\| \operatorname{IAS}\left(T_{0}\right)\right\rangle^{2} .
\end{gathered}
$$

Several estimates have been made for the reduced matrix element based on hydrodynamic and microscopic models ${ }^{49,7-9}$ ). Using the hydrodynamic model, Bohr, Damgaard and Mottelson ${ }^{49}$ ) derived the basic expression

$$
\mathscr{M}=K_{1} Z^{2} \text {. }
$$

Here, $K_{1} \approx 5 \times 10^{-3} \mathrm{MeV}^{2}$. In one form of a microscopic model ${ }^{7}$ ), the expression

$$
\mathcal{M}=K_{2} \frac{Z^{2}}{A^{4 / 3}}
$$

was obtained with $K_{2} \approx 2 \mathrm{MeV}^{2}$. It was not used in the present work because it resulted in inconsistencies. In another form of a microscopic model ${ }^{8}$ ), the expression

$$
\mathscr{M}=K_{3} \frac{Z^{2} N}{A^{2 / 3}}
$$

was obtained with $K_{3} \approx 5.3 \times 10^{-3} \mathrm{MeV}^{2}$.

A detailed matrix element has been derived by Lane and Mekjian ${ }^{9}$ ) by evaluating mixing effects of the charge monopole operator $c_{\mathrm{v}}$ in terms of sum rules of this operator. The result is

$$
M=\left\langle c_{\mathrm{v}}^{2}\right\rangle+\frac{T_{0}+1}{T_{0}}\left(L_{1}-L_{2}\right)+2 \frac{2 T_{0}+1}{2 T_{0}-1} L_{2} .
$$

Here, the leading term $\left\langle c_{\mathrm{v}}^{2}\right\rangle$ is the expectation value of $c_{\mathrm{v}}^{2}$ (which is proportional to $L_{0}$ ), and $L_{0}, L_{1}$, and $L_{2}$ are proportional to the isoscalar, isovector, and isotensor parts of $c_{\mathrm{v}} \times c_{\mathrm{v}}$. They depend on the moments $\left\langle r^{\lambda}\right\rangle_{N}$ and $\left\langle r^{\lambda}\right\rangle_{Z}$ with $\lambda=2$ and 4 of the distributions of neutron matter and proton matter (charge) in the nucleus. The results are

$$
\begin{aligned}
\left\langle c_{\mathrm{v}}^{2}\right\rangle & =2.46 \hbar \omega L_{0} / \bar{E}\left(T_{0}\right), \\
L_{0} & =\frac{1}{4} a_{1}^{2} A b^{2}\left\langle r^{2}\right\rangle_{A}, \\
L_{1} & =\frac{1}{4} a_{1}^{2}\left[\left(N\left\langle r^{4}\right\rangle_{N}-Z\left\langle r^{4}\right\rangle_{Z}-\frac{\left(N\left\langle r^{2}\right\rangle_{N}-Z\left\langle r^{2}\right\rangle_{Z}\right)^{2}}{N-Z}\right],\right. \\
L_{2} & =\frac{V_{1}}{A} \frac{T_{0}-\frac{1}{2}}{\bar{E}\left(T_{0}\right)} L_{1} .
\end{aligned}
$$


In these equations, $\hbar \omega$ and $b$ are the shell model energy and oscillator size parameters, respectively,

$$
\frac{\hbar^{2}}{m b^{2}}=\hbar \omega=41 A^{-1 / 3} \mathrm{MeV}
$$

The energy $\bar{E}\left(T_{0}\right)$ is the energy splitting between the $T_{0}$ component of the IVM and IAS. From eq. (5) it follows that

$$
\bar{E}\left(T_{0}\right)=E_{\mathrm{IVM}}\left(T_{0}\right)-E_{\mathrm{IAS}}\left(T_{0}\right)=V_{0} A^{-1 / 3}-V_{1} A^{-1} .
$$

The quantity $a_{1}$, finally, is

$$
a_{1}=\frac{1}{2}(Z-1) e^{2} / \tilde{R}^{3},
$$

where $\tilde{R}=R=\left[\frac{5}{3}\left\langle r^{2}\right\rangle_{Z}\right]^{1 / 2}$ is the equivalent charge radius of a uniform charge distribution. However, the authors ${ }^{9}$ ) argue that an increase of the value of $a_{1}$ is required resulting from a consistency condition on the Coulomb potential due to the nonuniformity of the nuclear charge distribution. This increase can be reproduced by decreasing the value of $\tilde{R}$ according to

$$
\tilde{R}=\left[\frac{5}{3}\left\langle r^{2}\right\rangle_{Z}\right]^{1 / 2}-0.15 \mathrm{fm} .
$$

Eqs. (25) to (33) implicitly define the square of the reduced matrix element $\mathscr{M}$ of eq. (21). An explicit expression is easily obtained in the approximations $\left\langle r^{\lambda}\right\rangle_{N}=$ $f\left\langle r^{\lambda}\right\rangle_{Z}$ with $f=1$ and $\tilde{R}=R$. The result using $V_{0}=155 \mathrm{MeV}$ and $V_{1}=55 \mathrm{MeV}$ is

$$
\begin{aligned}
\mathscr{M}= & \left(1.838 \times 10^{-2} \mathrm{MeV}^{2}\right) \frac{A^{4 / 3}}{\left\langle r^{2}\right\rangle_{Z}^{2}}(Z-1)^{2} \frac{c_{3}}{c_{2}} c_{1} \\
& \times\left\{1+\left[3.0462 \frac{T_{0}+1}{A^{4 / 3}} c_{2}-1.0811 \frac{\left(T_{0}+1\right)\left(T_{0}-\frac{1}{2}\right)}{A^{2}}+2.1621 \frac{\left(T_{0}\right)\left(T_{0}+\frac{1}{2}\right)}{A^{2}}\right]\right. \\
& \left.\times \frac{\left\langle r^{4}\right\rangle_{Z}-\left\langle r^{2}\right\rangle_{Z}^{2}}{\left\langle r^{2}\right\rangle_{Z}^{2}} \frac{c_{4}}{c_{3}}\right\},
\end{aligned}
$$

where $c_{1}=c_{2}=c_{3}=c_{4}=1$. The exact expression includes correction functions $c_{1}, c_{2}$, $c_{3}$, and $c_{4}$ (not given here) which are on the order of unity and can be expressed exactly as functions of $V_{0}, V_{1}, f,\left\langle r^{\lambda}\right\rangle_{Z}$, and $A$. The most important correction is

$$
c_{1}=\left[1-0.15 /\left(\frac{5}{3}\left\langle r^{2}\right\rangle_{Z}\right)^{1 / 2}\right]^{-6},
$$

which leads to an increase of about $20 \%$ as mentioned above. The exact equations have been used in the analysis presented in sect. 6 with several parametrizations of $\left\langle r^{\lambda}\right\rangle_{N}$ and $\left\langle r^{\lambda}\right\rangle_{Z}$

The expression for $\mathcal{M}$ of Lane and Mekjian ${ }^{9}$ ) has a functional form which is similar to the basic hydrodynamical expression of Bohr, Damgaard and Mottelson ${ }^{49}$ ) 
except that the magnitude is a factor of about 3 stronger. This result is obtained from eq. (34) with $\left\langle r^{2}\right\rangle^{1 / 2} \approx 0.93 A^{1 / 3} \mathrm{fm}, f=1, c_{1} \approx 1.2, c_{2}=c_{3}=c_{4}=1$, and \{\}$\approx 1.3$.

Eqs. (8) and (10) for $\Gamma^{\downarrow}$ in the strength-function approach can be related to the expression from the picket fence model, eq. (3). The approximate proportionality of both expressions to the density of the doorway states at $E_{\mathrm{IAS}}$ becomes apparent when $\Gamma_{\mathrm{IVM}}\left(E_{\mathrm{IVM}}\right)$ of eq. (11) is introduced. Assuming $\Delta E \equiv E_{\mathrm{IVM}}-E_{\mathrm{IAS}} \gg$ $\frac{1}{2} \Gamma_{\mathrm{IVM}}\left(E_{\mathrm{IAS}}\right)$, the result is $M_{\mathrm{AD}}^{2} \approx M_{\mathrm{AM}}^{2} M_{\mathrm{MD}}^{2} / \Delta E^{2}$. Here, $M_{\mathrm{AD}}, M_{\mathrm{AM}}$, and $M_{\mathrm{MD}}$ are again the matrix elements connecting respectively the IAS, doorway states, and the $\left(T_{0}-1\right)$ component of the IVM. The matrix elements $M_{\mathrm{AD}}$ and $M_{\mathrm{AM}}$ are chargedependent. The above equation leads to a value for $M_{\mathrm{AD}}$ which is approximately constant and on the order of $2 \mathrm{keV}$. This value was used in sect. 2 .

\section{Analysis and results}

\subsection{PRELIMINARY RESULTS}

Global least-squares analyses of the 65 spreading widths $\Gamma^{\downarrow}$ with $A>110$ of sect. 3 have been carried out using eq. (4) or eq. (8) with (10) and appropriate parameterizations of the charge-dependent matrix element $V_{\mathrm{CD}}=M_{\mathrm{AM}}$, the damping width $\Gamma_{\mathrm{IVM}}$, and the difference in excitation energies $E_{\mathrm{IVM}}-E_{\mathrm{IAS}}$. The use of these simple equations can only establish general trends for the parameters describing the IVM. Such information is very useful, though, given the scarcity of experimental knowledge about the IVM.

In an early analysis the hydrodynamic and microscopic matrix elements eqs. (18), (20), (22), and (24) were used as $V_{\mathrm{CD}}=\alpha V_{\mathrm{CD}}$ (theory) where $\alpha$ is an adjustable parameter. Furthermore, $V_{0}$ in eq. (6) was used as an adjustable parameter with $V_{1}$ fixed at $V_{1}=55 \mathrm{MeV}$. Most importantly, $\Gamma_{\mathrm{IVM}}$ was assumed to be constant and used as a third parameter. Good agreement with the data can be obtained. However, the parameters $\alpha, V_{0}$, and $\Gamma_{\text {IVM }}$ so obtained deviate strongly from the theoretical estimates and render the solutions meaningless.

When $V_{0}$ and $V_{1}$ of eq. (6) were fixed at reasonable values, $V_{0}=155 \mathrm{MeV}$ and $V_{1}=55 \mathrm{MeV}$, it became immediately apparent that the experimental spreading widths $\Gamma^{\downarrow}$ cannot be reproduced unless $\Gamma_{\mathrm{IVM}}$ is allowed to vary with mass number, isospin, and/or excitation energy $E_{\mathrm{IAS}}$. In one approach $\Gamma_{\mathrm{IVM}}$ was assumed to depend linearly on $A$ and $T$. Interestingly, a proportionality between $\Gamma_{\text {IVM }}$ and $T$ is sufficient, and the data can be reproduced very well in a two-parameter fit with a standard deviation of $\sigma \approx 12 \mathrm{keV}$ and $\chi^{2} / f=2.6$. The ratios $\alpha=V_{\mathrm{CD}} / V_{\mathrm{CD}}$ (theory) are expected to be on the order of unity, and indeed $\alpha \approx 1.4$ and 0.6 were observed for the hydrodynamic and microscopic matrix elements, respectively. However, a dependence $\Gamma_{\mathrm{IVM}}=\beta T$ with $\beta=1.0$ to $1.5 \mathrm{MeV}$ has no known theoretical justification. 
In another approach, eq. (11) for the distribution of coupling strength between the IVM and the doorway states, $\Gamma_{\mathrm{IVM}}(E)$, was parameterized by introducing the analytical expression

$$
\Gamma_{\mathrm{IVM}}(E)=\Gamma_{0} \Omega(E) / \Omega\left(E_{\mathrm{IVM}}\right) .
$$

If $\left\langle\right.$ IVM $\left|H_{\mathrm{MD}}\right|$ door $\rangle$ of eq. (11) is further assumed to be constant, then $\Omega(E) \propto$ $\rho_{\text {door }}(E)$. The parameterization which was used was found later to be quite appropriate for the low-energy tail near the IAS of the lorentzian line shape of $\Gamma_{\mathrm{IVM}}(E)$. The expression

$$
\Gamma_{0}=\left(2.3+72.3 A^{-2 / 3}\right) \mathrm{MeV}
$$

for $\Gamma_{0}=\Gamma_{\mathrm{IVM}}\left(E_{\mathrm{IVM}}\right)$ as obtained $\left.{ }^{59}\right)$ from a hydrodynamic model with nuclear viscosity was used in the analysis. Furthermore, the excitation energies $E_{\mathrm{IAS}}$ and $E_{\mathrm{IVM}}$ were replaced by the respective back-shifted energies $U$ to compensate for pairing effects as is done in the "back-shifted Fermi-gas formula" ${ }^{40-42}$ ). The functional form

$$
\Omega(E)=\text { const } U^{x}
$$

was introduced, and $\Gamma_{\mathrm{IVM}}\left(E_{\mathrm{IAS}}\right)$ was finally written as

$$
\Gamma_{\mathrm{IVM}}\left(E_{\mathrm{IAS}}\right)=\gamma\left(2.3+72.3 A^{-2 / 3}\right) \mathrm{MeV} \frac{U_{\mathrm{IAS}}^{x}}{U_{\mathrm{IVM}}^{x}} .
$$

The fit to the data contains the parameter $\alpha$ which represents the adjustment factor of the matrix elements, the parameter $\gamma$ which represents the adjustment factor for $\Gamma_{\mathrm{IVM}}\left(E_{\mathrm{IVM}}\right)$, and the exponent $x$ which describes the increase of the density of doorway states at low excitation energies.

A remarkably small value of $\chi^{2} / f \approx 2.0$ was obtained for the three-parameter fit with values of $\alpha \approx 1.2$ (hydrodynamic matrix element), $\gamma \approx 51$, and $x \approx 3.2$. Indeed, $\alpha$ is close to unity as expected. However, the large value of $\gamma$ points to a discrepancy which was later associated with the fact that the density of doorway states does not increase with excitation energy indefinitely but instead ${ }^{10}$ ) reaches a maximum value and decreases beyond. The exponent $x$ is in good agreement with theoretical estimates of $x \approx 3$ for the level densities of $2 p-2 h$ states in a uniform spacing model ${ }^{60,17}$ ). It is believed that the increase of the density of doorway states with excitation energy in the present analysis reflects, in part, the strong increase in deformation for several of the isotopic sequences contained as data input.

\subsection{THE STRENGTH-FUNCTION APPROACH}

A global least-squares analysis of the experimental spreading widths $\Gamma^{\downarrow}$ has been performed using the strength-function parameterization of MacDonald and Birse ${ }^{10}$ ). 
Three analytical expressions for the Coulomb matrix element $M_{\mathrm{AM}}$ were employed based on hydrodynamical, microscopic, and sum rule considerations, eq. (18) with eqs. (22), (24), and (25), respectively. The latter expression requires knowledge of the moments $\left\langle r^{\lambda}\right\rangle_{N}$ and $\left\langle r^{\lambda}\right\rangle_{Z}$ of the neutron and proton distributions. Three parameterizations were employed.

In one parameterization $(A)$, the radial moments of the neutron and proton density distributions were obtained from harmonic oscillator wave functions generated in identical potential wells as described in ref. $\left.{ }^{9}\right)$. The use of the same oscillator parameter $b$ for neutrons and protons implies $\left.\left\langle r^{2}\right\rangle_{N}\right\rangle\left\langle r^{2}\right\rangle_{Z}$ in heavy nuclei contrary to experimental evidence. It was argued ${ }^{9}$ ), however, that this is necessary to insure isospin purity of the unmixed state as required for such an analysis.

In another parameterization (B), the radial moments of the proton density distribution were obtained by numerical integration of a realistic Fermi distribution known to describe ${ }^{61}$ ) ground state charge distributions deduced from electron scattering $\left(r_{0}=1.115 A^{1 / 3}-0.53 A^{-1 / 3} \mathrm{fm}\right.$ and $\left.a=0.568 \mathrm{fm}\right)$. To guarantee isospin purity for the unmixed state [see ref. $\left.^{9}\right)$ ], the radial moments for neutrons and protons were assumed to satisfy

$$
\left\langle r^{\lambda}\right\rangle_{N}=(A / 2 Z)^{\lambda / 3}\left\langle r^{\lambda}\right\rangle_{Z}
$$

The ratio of the neutron and proton radial moments from eq. (40) is approximately equal to that from the harmonic oscillator wave functions.

The analytical expressions of Elton ${ }^{62}$ ) were used in a third parameterization (C). Here, Fermi charge distributions were assumed with constant charge density in the nuclear interior and constant diffuseness. The parameters were adjusted to describe electron scattering and muonic X-ray data. Evaluating the Fermi integrals up to fourth order ${ }^{63}$ ) yields for the moments of the charge distribution

$$
\begin{aligned}
\left\langle r^{2}\right\rangle_{Z}^{1 / 2} & =\left(\frac{3}{5}\right)^{1 / 2}\left(r_{0} A^{1 / 3}\right)\left\{1+\frac{5}{6} B^{2}-\frac{7}{24} B^{4}\right\}, \\
\left\langle r^{2}\right\rangle_{Z} & =\frac{3}{5}\left(r_{0} A^{1 / 3}\right)^{2}\left\{1+\frac{5}{3} B^{2}+\frac{1}{9} B^{4}\right\}, \\
\left\langle r^{4}\right\rangle_{Z} & =\frac{3}{7}\left(r_{0} A^{1 / 3}\right)^{4}\left\{1+\frac{14}{3} B^{2}+7 B^{4}\right\},
\end{aligned}
$$

with

$$
\begin{aligned}
& B=\frac{\pi a}{r_{0} A^{1 / 3}}, \\
& r_{0}=1.135 \mathrm{fm}, \\
& a=0.513 \mathrm{fm} .
\end{aligned}
$$

The moments of the neutron-matter distribution were parameterized as ${ }^{63}$ ) 


$$
\left\langle r^{\lambda}\right\rangle_{N}=f^{\lambda}\left\langle r^{\lambda}\right\rangle_{Z}
$$

with

$$
f=[(Z+(N-Z) 1.266) / N]^{1 / 2} .
$$

This expression is in good agreement with experimental data and the droplet-model. Improved parameterizations of nuclear charge distributions which include deformation effects have been reported ${ }^{64-66}$ ). The use of these expressions was not considered necessary in the present work.

A realistic analysis of the data in terms of the above equations should permit adjustments of certain quantities. These include the strength of the charge-dependent Coulomb matrix element $\alpha=V_{\mathrm{CD}} / V_{\mathrm{CD}}$ (calc.) and the energies $V_{0}$ and $V_{1}$ which describe the energy separation between IVM $\left(T_{0}-1\right)$ and IAS, eq. (6). Furthermore, the quantities $M_{20}$ and $x$ in $M_{2}=M_{20} A^{-x}$ of eq. (15) are the result of an adjustment to a very limited set of calculated values and may require further adjustments. The energies contained in eqs. (16) and (17), finally, may also have to be adjusted.

Fig. 6 displays the complete set of experimental data as a function of mass number A separately for each sequence of isotopes. The thin lines represent the result of

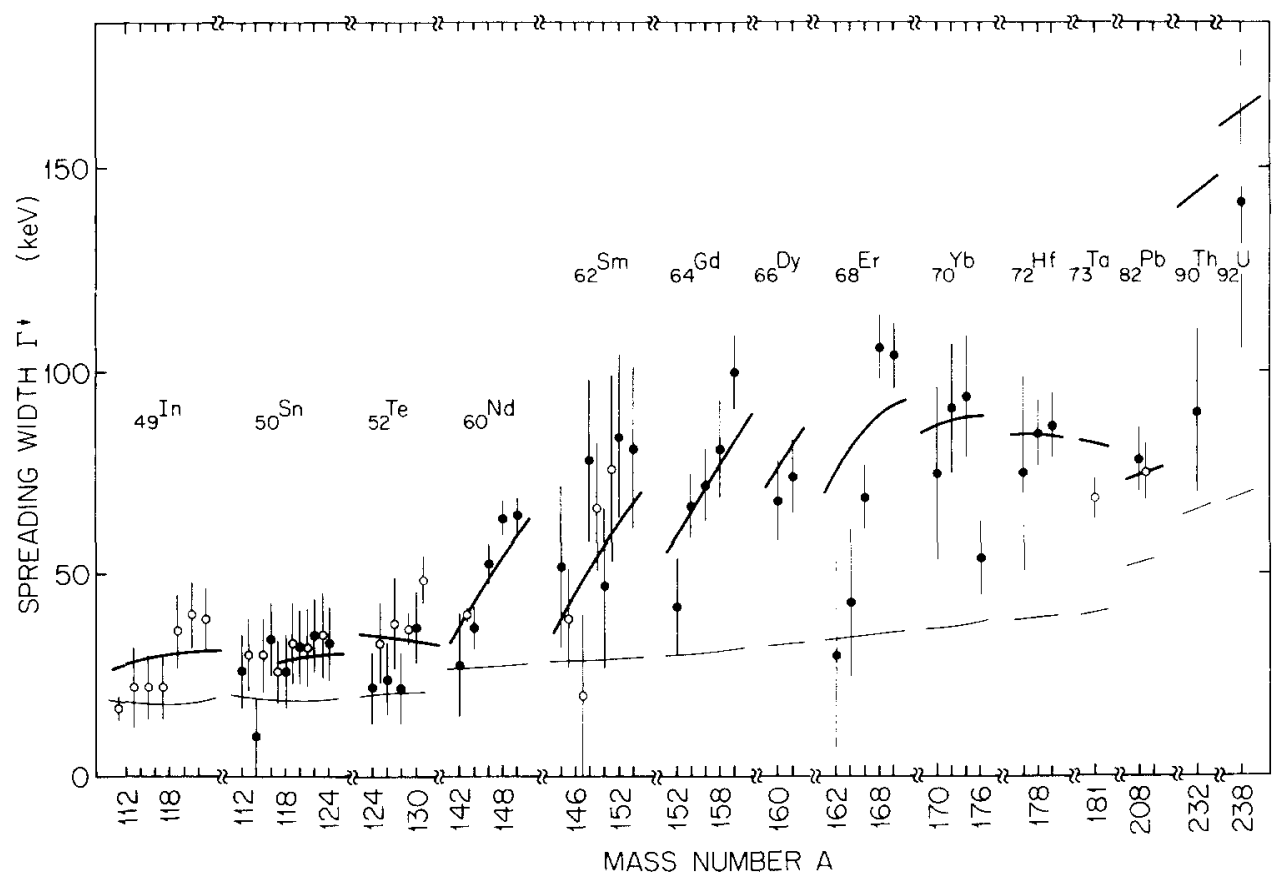

Fig. 6. Experimental spreading widths $\Gamma^{\downarrow}$ as function of mass number for the sequences of isobars with $Z \geqslant 49$. Total widths $\Gamma$ representing upper limits are shown for a number of nuclei (see text and table 2). The thin line is the result of unadjusted calculations with the sum rule matrix elements of Lane and Mekjian ${ }^{9}$ ), eqs. (19) and (25), and the strength function of MacDonald and Birse ${ }^{10}$ ), eq. (10) with eqs.

(12) to (17). The heavy line is from a fit with slightly adjusted quantities but including a dependence on deformation due to a postulated mixing with the IVQ (see text and case 3 of table 1). 
calculations using the above equations with unadjusted quantities. The measured values are underpredicted by factors of two to three. More importantly, the calculations do not reproduce the observed strong increases with neutron excess. No basic improvement could be achieved by adjusting parameters.

The solution to this problem was found ${ }^{67}$ ) in a postulated mixing in deformed nuclei between the giant isovector monopole (IVM) and the giant isovector quadrupole (IVQ) resonances. Similar effects are known for the respective isoscalar resonances ISM and ISQ (denoted in the literature usually by GMR and GQR). The ISM has been predicted ${ }^{68-70}$ ) to mix in deformed nuclei with the $\beta$-vibration component of ISQ. The latter resonance splits into $K=0$ ( $\beta$-vibration, $K=1$ and $K=2$ ( $\gamma$-vibration) components. The resulting splitting of the ISM strength has been observed experimentally for rare-earth nuclei ${ }^{71,72}$ ) and actinide nuclei ${ }^{73,74}$ ). Similar effects for the isovector resonances would be reflected in the second moment $M_{2}$ of the IVM strength function. In order to test this assumption, eq. (15) for $M_{2}$ was replaced by the simple parameterization

$$
M_{2} \approx M_{20} A^{-x}(1+y \beta)
$$

where $\beta$ represents the quadrupole deformation of the nuclear shape.

Experimental deformation parameters $\beta$ were used for some analyses, but a simplified treatment became possible with the use of the parameterization ${ }^{75}$ )

$$
\beta=\alpha_{0} n(1-n)(2-n) p(1-p)(2-p)+\alpha_{1} .
$$

Here, $n$ and $p$ are the relative neutron and proton occupation numbers within major shell regions. This equation reproduces the experimentally determined $\beta$-values rather well. The quantities $\alpha_{0}$ are shell-dependent ${ }^{76}$ ), and $\alpha_{1}$ displays a weak dependence on mass number $A$.

Including the quantity $y$ of eq. (49) as an adjustable parameter, several leastsquares analyses were performed. Strong correlations between some of the adjusted quantities were observed, and it was concluded that only two- or at most threeparameter fits with properly selected parameters lead to consistent results. The parameters established from these fits are listed in table 1 . The calculated spreading widths $\Gamma^{\downarrow}$ (case 3) are included in fig. 6 (heavy lines). The agreement between experimental and calculated widths is remarkably good. The values for $\chi^{2}$ per degree of freedom from table 1 are very small and range from about 1.2 to 1.3 for all fits using the sum rule matrix element (cases 3 to 5,8 to 10 , etc.). Slightly increased values are obtained with the hydrodynamic and microscopic matrix elements.

Adjusting only $V_{0}$ and $y$ (cases 3 to 5) leads to $V_{0} \approx 170 \mathrm{MeV}$ in excellent agreement with the estimate of Bohr et al. ${ }^{49}$ ). Accordingly, if $V_{0}=170 \mathrm{MeV}$ is assumed (cases 18 to 20 ), only a $\leqslant 2 \%$ adjustment of the sum rule matrix element is required. Adjusting both $V_{0}$ and $V_{1}$ (cases 8 to 10 ) gives $V_{1} \approx 65 \mathrm{MeV}$ which is probably too large. A fixed value $V_{1}=55 \mathrm{MeV}$ was assumed for all other cases. A value of $V_{0}=170 \mathrm{MeV}$ together with $\alpha=1$ (cases 28 to 30 ) leads furthermore to parameters for the second moment $M_{2}$ of eq. (59) in close agreement with the parameters (for 
TABLE 1

Parameters and $\chi^{2}$ per degree of freedom for fitting 65 experimental spreading widths $\Gamma^{\downarrow}$ for $A>110$

\begin{tabular}{|c|c|c|c|c|c|c|c|c|c|}
\hline Case & $\begin{array}{c}\text { Matrix } \\
\text { element }\end{array}$ & $\begin{array}{c}\text { Number } \\
\text { parameters }\end{array}$ & $\alpha$ & $\begin{array}{c}V_{0} \\
(\mathrm{MeV})\end{array}$ & $\begin{array}{c}V_{1} \\
(\mathrm{MeV})\end{array}$ & $\begin{array}{c}M_{20} \\
\left(\mathrm{MeV}^{2}\right)\end{array}$ & $x$ & $y$ & $\chi^{2} / f$ \\
\hline 1 & I & 2 & 1.00 & $102.5^{*}$ & 55.0 & 547.9 & 0.4558 & $2.93^{*}$ & 1.30 \\
\hline 2 & 11 & 2 & & $135.0^{*}$ & & & & $3.09^{*}$ & 1.43 \\
\hline 3 & IIIA & 2 & & $174.7^{*}$ & & & & $3.79^{*}$ & 1.21 \\
\hline 4 & IIIB & 2 & & $171.9^{*}$ & & & & $3.46^{*}$ & 1.22 \\
\hline 5 & IIIC & 2 & & $170.0^{*}$ & & & & $3.59^{*}$ & 1.27 \\
\hline 6 & I & 3 & 1.00 & $105.5^{*}$ & $61.5^{*}$ & 547.9 & 0.4558 & $2.82^{*}$ & 1.30 \\
\hline 7 & II & 3 & & $135.9^{*}$ & $56.8^{*}$ & & & $3.06^{*}$ & 1.45 \\
\hline 8 & IIIA & 3 & & $174.7^{*}$ & $55.0^{*}$ & & & $3.79^{*}$ & 1.23 \\
\hline 9 & IIIB & 3 & & $175.1^{*}$ & $62.6^{*}$ & & & $3.32^{*}$ & 1.23 \\
\hline 10 & IIIC & 3 & & $175.4^{*}$ & $69.2^{*}$ & & & $3.33^{*}$ & 1.25 \\
\hline 11 & I & 2 & $2.31^{*}$ & 155.0 & 55.0 & 547.9 & 0.4558 & $4.20^{*}$ & 1.57 \\
\hline 12 & II & 2 & $1.33^{*}$ & & & & & $3.48^{*}$ & 1.45 \\
\hline 13 & IIIA & 2 & $0.75^{*}$ & & & & & $3.44^{*}$ & 1.22 \\
\hline 14 & IIIB & 2 & $0.78^{*}$ & & & & & $3.22^{*}$ & 1.22 \\
\hline 15 & IIIC & 2 & $0.80^{*}$ & & & & & $3.38^{*}$ & 1.25 \\
\hline 16 & I & 2 & $2.79^{*}$ & 170.0 & 55.0 & 547.9 & 0.4558 & $4.59^{*}$ & 1.66 \\
\hline 17 & II & 2 & $1.60^{*}$ & & & & & $3.76^{*}$ & 1.49 \\
\hline 18 & IIIA & 2 & $0.94^{*}$ & & & & & $3.70^{*}$ & 1.21 \\
\hline 19 & IIIB & 2 & $0.97^{*}$ & & & & & $3.45^{*}$ & 1.22 \\
\hline 20 & IIIC & 2 & $1.00^{*}$ & & & & & $3.63^{*}$ & 1.27 \\
\hline 21 & I & 3 & 1.00 & 155.0 & 55.0 & $1000.0^{*}$ & $0.3000^{*}$ & $1.94^{*}$ & 1.53 \\
\hline 22 & II & 3 & & & & $1000.0^{*}$ & $0.4767^{*}$ & $2.94^{*}$ & 1.46 \\
\hline 23 & IIIA & 3 & & & & $900.2^{*}$ & $0.6702^{*}$ & $4.43^{*}$ & 1.16 \\
\hline 24 & IIIB & 3 & & & & $805.9^{*}$ & $0.6316^{*}$ & $3.97^{*}$ & 1.20 \\
\hline 25 & IIIC & 3 & & & & $598.8^{*}$ & $0.5611^{*}$ & $4.00^{*}$ & 1.27 \\
\hline 26 & I & 3 & 1.00 & 170.0 & 55.0 & $1000.0^{*}$ & $0.2415^{*}$ & $1.72^{*}$ & 1.65 \\
\hline 27 & II & 3 & & & & $1000.0^{*}$ & $0.4083^{*}$ & $2.68^{*}$ & 1.52 \\
\hline 28 & IIIA & 3 & & & & $1000.0^{*}$ & $0.6057^{*}$ & $4.15^{*}$ & 1.18 \\
\hline 29 & IIIB & 3 & & & & $1000.0^{*}$ & $0.5903^{*}$ & $3.73^{*}$ & 1.21 \\
\hline 30 & IIIC & 3 & & & & $815.2^{*}$ & $0.5382^{*}$ & $3.76^{*}$ & 1.28 \\
\hline
\end{tabular}

The charge-dependent Coulomb matrix elements are $\mathrm{I}=$ hydrodynamic, $\mathrm{II}=$ microscopic, $1 \mathrm{II}=\mathrm{sum}$ rule. $\mathrm{A}, \mathrm{B}$, and $\mathrm{C}$ denote the three methods of parameterizing the radial moments $\left\langle r^{2}\right\rangle$ and $\left\langle r^{4}\right\rangle$ discussed in the text. Adjusted quantities are marked by *.

The parameters denote $\alpha=V_{\mathrm{CD}} / V_{\mathrm{CD}}$ (theory), $E_{\mathrm{IVM}}\left(T_{0}-1\right)-E_{\mathrm{IAS}}\left(T_{0}\right)=V_{0} A^{-1 / 3}-\left(T_{0}+1\right) V_{1} A^{-1}$, $M_{2}=M_{20} A^{-x}(1+y \beta)$. An upper bound of $M_{20}=1000 \mathrm{MeV}^{2}$ was used.

$\beta=0)$ introduced earlier $\left.{ }^{10}\right)$. The widths $\Gamma_{\mathrm{IVM}}\left(E_{\mathrm{IVM}}\right)$ and $\Gamma_{\mathrm{IVM}}\left(E_{\mathrm{IAS}}\right)$ obtained from the second moments $M_{2}$ of the IVM strength function (case 28) are displayed in fig. 7 for nuclei along the line of $\beta$-stability. The curves are derived for both, $\beta \neq 0$ and $\beta=0$.

The above considerations in favor of $V_{0} \approx 170 \mathrm{MeV}$, however, are not in full agreement with the results from the RPA calculations ${ }^{52}$ ) which favor $V_{0} \approx 155 \mathrm{MeV}$ 


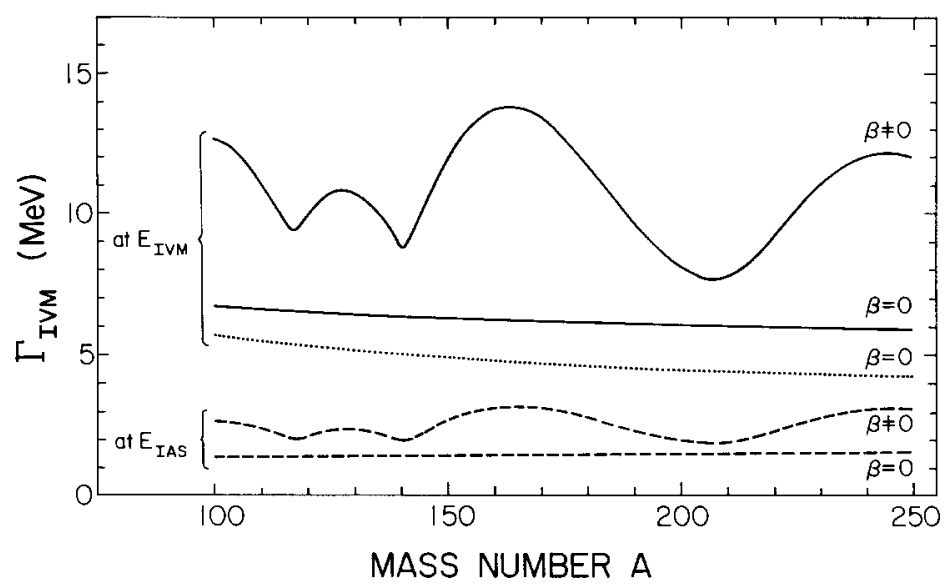

Fig. 7. Calculated widths of $\Gamma_{\mathrm{IVM}}$ at the energies $E_{\mathrm{IVM}}$ (solid lines) and $E_{\mathrm{IAs}}$ (dashed line) from eqs. (12) and (49) both with and without the inclusion of deformation effects for nuclei along the line of $\beta$-stability. Predictions assuming compression modes in a hydrodynamic model with viscosity ${ }^{59}$ ) are included as dotted line.

(see also fig. 3). If $V_{0} \approx 155 \mathrm{MeV}$ is assumed (cases 13 to 15 ), the data require a reduction by a factor of $\alpha \approx 0.79$ of the sum rule matrix element. Such an adjustment reverses almost quantitatively the adjustment of eqs. (33) and (35) introduced phenomenologically by Lane and Mekjian ${ }^{9}$ ). Alternately, if $\alpha=1$ is assumed (cases 23 to 25 ), the parameters which describe the second moment $M_{2}$ and hence the width $\Gamma_{\text {IVM }}\left(E_{\text {IVM }}\right.$ ) lead to values which are a factor of about 0.65 smaller (for $\beta \equiv 0$ ) than those introduced by MacDonald and Birse ${ }^{10}$ ). However, they are in almost perfect agreement with the widths $\Gamma_{\text {IVM }}$ calculated on the basis of compression modes in a hydrodynamic model with viscosity ${ }^{59}$ ), eq. (37). These hydrodynamic estimates are included in fig. 7.

It appears that the present analysis cannot distinguish between the above solutions. The extremely limited experimental information on excitation energies and widths of the IVM does, of course, not simplify any judgement.

The parameter $y$ which accounts for the dependence on deformation has consistent values in the range 3.3 to 4.1 with an average of $y \approx 3.7$. This means that the experimentally observed width $\Gamma_{\text {IVM }}$ doubles for a nucleus with a deformation of $\beta \approx 0.27$.

The hydrodynamic and microscopic matrix elements of eqs. (22) and (24) consistently underestimate the data. Comparing the results of the cases 16 to 20 suggests that the two matrix elements should be increased by factors of about 2.8 and 1.6, respectively.

Excellent agreement exists between the calculated and experimental reduced widths $\Gamma^{\downarrow}$ as pointed out above and displayed in fig. 6 . However, a few shortcomings are also apparent. The values for ${ }^{111} \mathrm{In}$ and ${ }^{176} \mathrm{Yb}$ deviate strongly from the predicted trends and have, in fact, been excluded from the least-squares analyses. The low 
experimental value for ${ }^{176} \mathrm{Yb}$ has been questioned before ${ }^{13}$ ) but no explanation could be found. While the strong increase in $\Gamma^{\downarrow}$ with neutron excess is well reproduced for most of the rare-earth isotopic sequences, it appears that the experimental increases for $Z=64$ and particularly $Z=68$ are stronger than calculated. The high experimental values for the neutron-rich members may represent overestimates (see sect. 3), but the low values for the neutron-deficient members cannot be explained. Some additional isospin dependence may therefore exist. The observed ${ }^{12}$ ) increase for $Z=49$ appears to result from contributions of 0 to $18 \mathrm{keV}$ from the escape width $\Gamma^{\uparrow}$ which were not included in the original analysis of the data ${ }^{12}$ ). The overall agreement is truly remarkable. It would be desirable to compare additional data points for the actinide nuclei with the calculations.

\section{Summary}

A global least-squares analysis of 65 experimental spreading widths $\Gamma^{\downarrow}$ of isobaric analog states with $A>110$ leads to excellent agreement with a standard deviation between experimental and calculated values of about $7 \mathrm{keV}$ and $\chi^{2}$ per degree of freedom of 1.2 to 1.3 . Mixing with $1 \mathrm{p}-1 \mathrm{~h}$ and $2 \mathrm{p}-2 \mathrm{~h}$ doorway states of lower isospin is assumed to be mediated by coupling to the $\left(T_{0}-1\right)$ component of the giant isovector monopole resonance. The density of these doorway states near the isobaric analog states is much lower than the density of all states with the same spin and parity.

The strength-function approach of MacDonald and Birse ${ }^{10}$ ) was used, but a splitting of the isovector monopole strength in deformed nuclei due to coupling to the isovector quadrupole resonance has to be assumed to describe the data. The global dependences on mass number $A$, on nuclear size and shape and on the parameters describing the isovector monopole resonance have been established. These quantities were found in acceptable agreement with various theoretical estimates and with the restricted experimental information. The strength of the chargedependent matrix element responsible for the isospin admixtures in isobaric analog states of heavy nuclei was found to be in very good agreement with an expression derived from sum rules ${ }^{9}$ ) but slightly stronger than hydrodynamic ${ }^{49}$ ) and microscopic $^{8}$ ) estimates.

Discussions with K.T. Hecht are greatly appreciated. This work was supported in part by the National Science Foundation, Grant No. PHY-8308072 and the Stichting voor Fundamenteel Onderzoek der Materie (FOM) which is financially supported by the Nederlandse Organisatie voor Zuiver Wetenschappelijk Onderzoek (ZWO).

\section{Appendix}

Table 2 contains a listing of the experimental spreading widths $\Gamma^{\downarrow}$ used as data base. Weighted averages are given in a few cases. 
TABLE 2

Experimental spreading widths $\Gamma^{\downarrow}$ and excitation energies $E_{\mathrm{IAS}}$ of isobaric analog states with $A>110$

\begin{tabular}{|c|c|c|c|c|c|c|}
\hline \multirow{2}{*}{$\begin{array}{c}\text { Isobaric } \\
\text { pair }\end{array}$} & \multicolumn{2}{|c|}{ Parent nucleus } & \multirow{2}{*}{$\begin{array}{c}E_{\mathrm{IAS}} \\
(\mathrm{MeV})\end{array}$} & \multirow{2}{*}{$J^{\pi}$} & \multirow{2}{*}{$\left.\Gamma^{\downarrow \mathrm{x}}\right)$} & \multirow{2}{*}{ Ref. } \\
\hline & $Z$ & $A$ & & & & \\
\hline \multirow[t]{7}{*}{$\mathrm{Sb}-\mathrm{Sn}$} & 50 & 112 & 6.409 & $0^{+}$ & $26.0 \pm 9.0$ & a) \\
\hline & 50 & 114 & 7.468 & $0^{+}$ & $10.0 \pm 9.0$ & a) \\
\hline & 50 & 116 & 8.483 & $0^{+}$ & $34.0 \pm 9.0$ & a) \\
\hline & 50 & 118 & 9.320 & $0^{+}$ & $26.0 \pm 9.0$ & $a, b)$ \\
\hline & 50 & 120 & 10.267 & $0^{+}$ & $32.0 \pm 9.0$ & $a, b)$ \\
\hline & 50 & 122 & 11.262 & $0^{+}$ & $35.0 \pm 9.0$ & a) \\
\hline & 50 & 124 & 12.187 & $0^{+}$ & $33.0 \pm 9.0$ & a) \\
\hline \multirow[t]{4}{*}{ I-Te } & 52 & 124 & 10.159 & $0^{+}$ & $22.0 \pm 9.0$ & a) \\
\hline & 52 & 126 & 11.101 & $0^{+}$ & $24.0 \pm 9.0$ & a) \\
\hline & 52 & 128 & 11.945 & $0^{+}$ & $22.0 \pm 9.0$ & a) \\
\hline & 52 & 130 & 12.704 & $0^{+}$ & $37.0 \pm 9.0$ & a) \\
\hline \multirow[t]{5}{*}{ Pm-Nd } & 60 & 142 & 9.976 & $0^{+}$ & $27.5 \pm 12.5$ & c) \\
\hline & 60 & 144 & 12.398 & $0^{+}$ & $36.7 \pm 5.3$ & c) \\
\hline & 60 & 146 & 13.147 & $0^{+}$ & $52.4 \pm 5.0$ & c) \\
\hline & 60 & 148 & 14.000 & $0^{+}$ & $63.8 \pm 4.3$ & c) \\
\hline & 60 & 150 & 14.337 & $0^{+}$ & $64.6 \pm 4.6$ & c) \\
\hline \multirow[t]{5}{*}{ Eu-Sm } & 62 & 144 & 8.996 & $0^{+}$ & $\leqslant 52.0 \pm 20.0$ & d) \\
\hline & 62 & 148 & 11.974 & $0^{+}$ & $\leqslant 78.0 \pm 20.0$ & d) \\
\hline & 62 & 150 & 12.672 & $0^{+}$ & $\leqslant 47.0 \pm 20.0$ & d) \\
\hline & 62 & 152 & 13.028 & $0^{+}$ & $\leqslant 84.0 \pm 20.0$ & d) \\
\hline & 62 & 154 & 14.103 & $0^{+}$ & $\leqslant 81.0 \pm 20.0$ & d) \\
\hline \multirow[t]{5}{*}{ Tb-Gd } & 64 & 152 & 11.552 & $0^{+}$ & $\leqslant 42.0 \pm 12.0$ & e) \\
\hline & 64 & 154 & 11.865 & $0^{+}$ & $\leqslant 67.0 \pm 8.0$ & e) \\
\hline & 64 & 156 & 12.823 & $0^{+}$ & $\leqslant 72.0 \pm 9.0$ & e) \\
\hline & 64 & 158 & 13.970 & $0^{+}$ & $\leqslant 81.0 \pm 12.0$ & e) \\
\hline & 64 & 160 & 15.022 & $0^{+}$ & $\leqslant 100.0 \pm 9.0$ & e) \\
\hline \multirow[t]{2}{*}{ Ho-Dy } & 66 & 160 & 12.324 & $0^{+}$ & $\leqslant 68.0 \pm 10.0$ & e) \\
\hline & 66 & 162 & 13.432 & $0^{+}$ & $\leqslant 74.0 \pm 9.0$ & e) \\
\hline \multirow[t]{5}{*}{ Tm-Er } & 68 & 162 & 11.283 & $0^{+}$ & $\leqslant 30.0 \pm 23.0$ & e) \\
\hline & 68 & 164 & 12.043 & $0^{+}$ & $\leqslant 43.0 \pm 18.0$ & e) \\
\hline & 68 & 166 & 12.917 & $0^{+}$ & $\leqslant 69.0 \pm 8.0$ & $\left.{ }^{e}\right)$ \\
\hline & 68 & 168 & 14.214 & $0^{+}$ & $\leqslant 106.0 \pm 8.0$ & e) \\
\hline & 68 & 170 & 15.493 & $0^{+}$ & $\leqslant 104.0 \pm 8.0$ & e) \\
\hline \multirow[t]{4}{*}{$\mathrm{Lu}-\mathrm{Yb}$} & 70 & 170 & 12.874 & $0^{+}$ & $\leqslant 75.0 \pm 21.0$ & e) \\
\hline & 70 & 172 & 13.728 & $0^{+}$ & $\leqslant 91.0 \pm 16.0$ & e) \\
\hline & 70 & 174 & 14.801 & $0^{+}$ & $\leqslant 94.0 \pm 15.0$ & e) \\
\hline & 70 & 176 & 16.023 & $0^{+}$ & $\left.\leqslant 54.0 \pm 9.0^{y}\right)$ & e) \\
\hline \multirow[t]{3}{*}{ Ta-Hf } & 72 & 176 & 13.508 & $0^{+}$ & $\leqslant 75.0 \pm 24.0$ & $\left.{ }^{e}\right)$ \\
\hline & 72 & 178 & 14.660 & $0^{+}$ & $\leqslant 85.0 \pm 8.0$ & e) \\
\hline & 72 & 180 & 15.659 & $0^{+}$ & $\leqslant 87.0 \pm 8.0$ & e) \\
\hline $\mathrm{Bi}-\mathrm{Pb}$ & 82 & 208 & 15.172 & $0^{+}$ & $78.0 \pm 8.0$ & $f, g)$ \\
\hline Pr-Th & 90 & 232 & 18.480 & $0^{+}$ & $90.0 \pm 20.0$ & h) \\
\hline
\end{tabular}


TABLE 2 (cont.)

\begin{tabular}{|c|c|c|c|c|c|c|}
\hline \multirow{2}{*}{$\begin{array}{c}\text { Isobaric } \\
\text { pair }\end{array}$} & \multicolumn{2}{|c|}{ Parent nucleus } & \multirow{2}{*}{$\begin{array}{c}E_{\mathrm{IAS}} \\
(\mathrm{MeV})\end{array}$} & \multirow{2}{*}{$J^{\pi}$} & \multirow{2}{*}{$\Gamma^{\downarrow}$} & \multirow{2}{*}{ Ref. } \\
\hline & $Z$ & $A$ & & & & \\
\hline $\mathrm{Np}-\mathrm{U}$ & 92 & 238 & 19.090 & $0^{+}$ & $\leqslant 142.0 \pm 37.0$ & h) \\
\hline \multirow[t]{7}{*}{ Sn-In } & 49 & 111 & 10.507 & $\frac{9}{2}^{+}$ & $\left.\leqslant 17.0 \pm 3.0^{y}\right)$ & i) \\
\hline & 49 & 113 & 11.826 & $\frac{9}{2}+$ & $\leqslant 22.0+10.0$ & i) \\
\hline & 49 & 115 & 13.317 & $\frac{2}{2}+$ & $\leqslant 22.0 \pm 8.0$ & i) \\
\hline & 49 & 117 & 14.151 & $\frac{9}{2}+$ & $\leqslant 22.0 \pm 8.0$ & i) \\
\hline & 49 & 119 & 14.995 & $\frac{9}{2}+$ & $\leqslant 36.0 \pm 9.0$ & i) \\
\hline & 49 & 121 & 15.953 & $\frac{9}{2}+$ & $\leqslant 40.0 \pm 8.0$ & i) \\
\hline & 49 & 123 & 16.943 & $\frac{\bar{q}}{2}+$ & $\leqslant 39.0 \pm 8.0$ & i) \\
\hline \multirow[t]{6}{*}{$\mathrm{Sb}-\mathrm{Sn}$} & 50 & 113 & 9.227 & $\frac{1}{2}^{+}$ & $30.0 \pm 9.0$ & b) \\
\hline & 50 & 115 & 10.084 & $\frac{2}{2}+$ & $30.0 \pm 9.0$ & b) \\
\hline & 50 & 117 & 11.283 & $\frac{1}{2}^{2}$ & $26.0 \pm 7.8$ & b) \\
\hline & 50 & 119 & 12.362 & $\frac{1}{2}^{+}$ & $33.0 \pm 9.9$ & b) \\
\hline & 50 & 121 & 13.285 & $\frac{2}{2}^{2}+$ & $32.0 \pm 9.6$ & b) \\
\hline & 50 & 123 & 14.228 & $\frac{2}{2}+$ & $35.0 \pm 10.5$ & b) \\
\hline \multirow[t]{4}{*}{$\mathrm{I}-\mathrm{Te}$} & 52 & 125 & 13.105 & $\frac{1}{2}^{+}$ & $33.0 \pm 9.9$ & i) \\
\hline & 52 & 127 & 13.923 & $\frac{3}{2}+$ & $38.0 \pm 11.4$ & i) \\
\hline & 52 & 129 & 14.671 & $\frac{3}{2}+$ & $37.0 \pm 4.0$ & $j, k_{j}$ \\
\hline & 52 & 131 & 15.370 & $\frac{3}{2}+$ & $49.0 \pm 6.0$ & $\mathrm{i}, \mathrm{k}, \mathrm{l}, \mathrm{m}, \mathrm{m})$ \\
\hline $\mathrm{Pm}-\mathrm{Nd}$ & 60 & 143 & 13.740 & $\frac{7-}{2}$ & $40.0 \pm 1.2$ & $\left.{ }^{k}\right)$ \\
\hline \multirow[t]{4}{*}{$\mathrm{Eu}-\mathrm{Sm}$} & 62 & 145 & 12.500 & $\frac{7-}{2}-$ & $39.0 \pm 12.0$ & 1) \\
\hline & 62 & 147 & 13.374 & $\frac{7}{2}-$ & $20.0 \pm 20.0$ & 1 \\
\hline & 62 & 149 & 14.331 & $\frac{7}{2}$ & $67.0 \pm 16.0$ & ) \\
\hline & 62 & 151 & 15.006 & $\frac{5}{2}^{-}$ & $76.0 \pm 23.0$ & 1) \\
\hline W-Ta & 73 & 181 & 16.573 & $\frac{7}{2}^{+}$ & $69.0 \pm 5.0$ & m) \\
\hline $\mathrm{Bi}-\mathrm{Pb}$ & 82 & 209 & 18.626 & $\frac{9}{2}^{+}$ & $75.0 \pm 7.0$ & g) \\
\hline
\end{tabular}

${ }^{\text {a) }}$ Ref. ${ }^{21}$ ). ${ }^{\text {b) }}$ Ref. ${ }^{26}$ ). ${ }^{\text {) }}$ Ref. ${ }^{17}$ ). ${ }^{\text {) }}$ Ref. ${ }^{14}$ ). ${ }^{\text {}}$ ) Ref. ${ }^{13}$ ). ${ }^{\text {f) }}$ Ref. ${ }^{38}$ ). ${ }^{8}$ ) Ref. ${ }^{18}$ ). ${ }^{\text {b) Ref. }}{ }^{11}$ ). ${ }^{1}$ ) Ref. ${ }^{12}$ ). $\left.{ }^{5}\right)$ Ref. ${ }^{30}$ ). ${ }^{\text {k) }}$ Ref. ${ }^{32}$ ). ${ }^{1}$ ) Ref. ${ }^{34}$ ). ${ }^{\text {m) }}$ Ref. ${ }^{37}$ ).

${ }^{\mathrm{x}}$ ) Total widths $\Gamma$ are listed for all even- $A$ isotopes from Sm to $\mathrm{Hf}$ and for the odd- $A$ isotopes of In. These values are preceded by the $\leqslant$ sign. The spreading widths $\Gamma^{\star}$ for the neutron-rich isotopes may be about $30 \%$ smaller (e.g., ${ }^{160} \mathrm{Gd},{ }^{168,170} \mathrm{Er}$; see text).

$\left.{ }^{y}\right)$ Not included in least-squares adjustments.

\section{References}

1) D.H. Wilkinson, Isospin in nuclear physics (North-Holland, Amsterdam, 1969)

2) N. Auerbach, Phys. Reports 98 (1983) 273

3) H.A. Weidenmüller, Nucl. Phys. A99 (1967) 269 and 289

4) A.M. Lane, in Isospin in nuclear physics, ed. D.H. Wilkinson (North-Holland, Amsterdam, 1969) ch. 11, p. 509

5) A.Z. Mekjian, Nucl. Phys. A146 (1970) 288

6) A.Z. Mekjian, Phys. Rev. Lett. 25 (1970) 888

7) N. Auerbach, J. Hüfner, A.K. Kerman and C.M. Shakin, Rev. Mod. Phys. 44 (1972) 48

8) N. Auerbach, Nucl. Phys. A182 (1972) 247

9) A.M. Lane and A.Z. Mekjian, Adv. Nucl. Phys. 7 (1973) 97 
10) W.M. MacDonald and M.C. Birse, Phys. Rev. C29 (1984) 425

11) S.Y. van der Werf, N. Blasi, S. Brandenburg, A.G. Drentje, M.N. Harakeh, W.A. Sterrenburg, B. Visscher, A. van der Woude, R. De Leo and H. Janszen, Phys. Lett. 105B (1981) 111

12) H. Taketani, M. Adachi, T. Matsuzaki, M. Matoba, N. Koori, T. Yamazaki, S. Morinobu, I. Katayama, M. Fujiwara, Y. Fujita and H. Ikegami, Phys. Lett. 90B (1980) 214;

M. Tohyama, Nucl. Phys. A401 (1983) 211

13) J. Jänecke, E.H.L. Aarts, A.G. Drentje, M.N. Harakeh and C. Gaarde, Nucl. Phys. A394 (1983) 39

14) J. Jänecke, F.D. Becchetti, W.S. Gray, R.S. Tickle and E. Sugarbaker, Nucl. Phys. A402 (1983) 262

15) M.L. Cescato, M.C. Hermida, M. Ruiz, J.L. Foster, Jr. and F. Krmpotic, Phys. Rev. C29 (1984) 49

16) M.C. Hermida, M. Ruiz, M.L. Cescato, J.L. Foster, Jr. and F. Krmpotic, Phys. Rev. C29 (1984) 64

17) H.J. Hofmann, S. Brandenburg, P. Grasdijk, M.N. Harakeh, W.A. Sterrenburg and S.Y. van der Werf, Nucl. Phys. A433 (1985) 181

18) R. Melzer, P. von Brentano and H. Paetz Gen. Schieck, Nucl. Phys. A432 (1985) 363;

G. Latzel and H. Paetz Gen. Schieck, Nucl. Phys. A323 (1979) 413

19) P.S. Miller and G.T. Garvey, Nucl. Phys. A163 (1971) 65

20) G.A. Whitten, Jr., J. Chai, N. Chirapatpimol, W.H. Dunlop and G. Igo, Phys. Lett. 51B (1974) 45

21) F.D. Becchetti, W.S. Gray, J. Jänecke, E.R. Sugarbaker and R.S. Tickle, Nucl. Phys. A271 (1976) 77

22) J.D. Bowman, H.W. Baer, R. Bolton, M.D. Cooper, F.H. Cverna, N.S.P. King, M. Leitch, H.S. Matis, J. Alster, A. Doron, M.A. Moinester, E. Blackmore and E.R. Siciliano, Phys. Rev. Lett. 50 (1983) 1195

23) H.W. Baer, R. Bolton, J.D. Bowman, M.D. Cooper, F.H. Cverna, N.S.P. King, M. Leitch, H.S. Matis, J. Alster, A. Doron, A. Erell, M.A. Moinester, E. Blackmore and E.R. Siciliano, Nucl. Phys. A396 (1983) 437c

24) A. Erell, J. Alster, J. Lichtenstadt, M.A. Moinester, J.D. Bowman, M.D. Cooper, F. Irom, H.S. Matis, E. Piasetzky, U. Sennhauser and Q. Ingram, Phys. Rev. Lett. 52 (1984) 2134

25) A. Erell, J. Alster, J. Lichtenstadt, M.A. Moinester, J.D. Bowman, H.W. Baer, M.D. Cooper, F. Irom, H.S. Matis, E. Piasetzky and U. Sennhauser, Phys. Rev. C, to be published

26) P. Richard, C.F. Moore, J.A. Becker and J.D. Fox, Phys. Rev. 145 (1966) 971

27) D.D. Long, P. Richard, C.F. Moore and J.D. Fox, Phys. Rev. 149 (1966) 906

28) G. Clausnitzer, R. Fleischmann, G. Graw, D. Proetel and J.P. Wurm, Nucl. Phys. A106 (1968) 99

29) R.K. Jolly and C.F. Moore, Phys. Rev. 155 (1967) 1377

30) J.L. Foster Jr., P.J. Riley and C.F. Moore, Phys. Rev. 175 (1968) 1498

31) K. Mudersbach, A. Heusler and J.P. Wurm, Nucl. Phys. A146 (1970) 477

32) E. Grosse, K. Melchior, H. Seitz, P. Von Brentano, J.P. Wurm and S.A.A. Zaidi, Nucl. Phys. A142 (1970) 345

33) J. Burde, G. Engler, A. Ginsburg, A.A. Jaffe, A. Marinov and L. Birstein, Nucl. Phys. A141 (1970) 375

34) H.R. Hiddleston, C.L. Hollas, V.D. Mistry and P.J. Riley, Phys. Rev. C3 (1971) 905

35) N.H. Merrill, S.W. Whineray, W.M. Zuk, D.C. Weisser, C.L. Hollas and M. Borsaru, Nucl. Phys. A216 (1973) 61

36) S. Gales, Y. El Hage, J.P. Schapira, S. Fortier, H. Laurent and J.M. Maison, Phys. Rev. C21 (1980) 98

37) G.W.R. Leibbrandt, et al., to be published; report KVI-115i (1986)

38) C. Gaarde, J.S. Larsen, A.G. Drentje, M.N. Harakeh and S.Y. van der Werf, Phys. Rev. Lett. 46 (1981) 902

39) M.N. Harakeh, Proc. Int. Symp. Nuclear fission and related collective phenomena and properties of heavy nuclei, Bad Honnef, Germany (1981); Lecture notes in Physics 158 (1982) 236

40) A. Gilbert and A.G.W. Cameron, Can. J. Phys. 43 (1965) 1446

41) W. Dilg, W. Schantl, H. Vonach and M. Uhl, Nucl. Phys. A217 (1973) 269

42) J.A. Holmes, S.E. Woosley, W.A. Fowler and B.A. Zimmerman, At. Data and Nucl. Data Tables 18 (1976) 305

43) E. Kuhlmann, Phys. Rev. C20 (1979) 415

44) E. Kuhlmann, Z. Phys. A322 (1985) 527

45) H.L. Harney, A. Richter and H.A. Weidenmüller, Rev. Mod. Phys. 58 (1986) 607 
46) D. Boose, H.L. Harney and H.A. Weidenmüller, Phys. Rev. Lett. 56 (1986) 2012

47) R.J. Blin-Stoyle, in Isospin in nuclear physics, ed. D.H. Wilkinson (North-Holland, Amsterdam, 1969) ch. 4, p. 115

48) A. Bohr and B. Mottelson, Nuclear structure, vol. I (Benjamin, New York, 1969) and vol. II (Benjamin, Reading, Massachusetts, 1975)

49) A. Bohr, J. Damgaard and B.R. Mottelson, Nuclear structure, ed. A. Hossain et al. (North-Holland, Amsterdam 1967), p. 1

50) R.ö. Akyüz and S. Fallieros, Phys. Rev. Lett. 27 (1971) 1016

51) N. Auerbach and A. Yeverechyahu, Nucl. Phys. A332 (1979) 173

52) N. Auerbach and A. Klein, Nucl. Phys. A395 (1983) 77

53) G.F. Bertsch and S.F. Tsai, Phys. Reports 18C (1975) 126

54) K.F. Liu and G.E. Brown, Nucl. Phys. A265 (1976) 385

55) N. Auerbach and N. Van Giai, Phys. Lett. 72B (1978) 289

56) N. Auerbach, V. Bernard and N. Van Giai, Nucl. Phys. A337 (1980) 143

57) S. Adachi and S. Yoshida, Nucl. Phys. A306 (1978) 53

58) K. Goecke, B. Castel and P.-G. Rheinhard, Nucl. Phys. A339 (1980) 377

59) N. Auerbach and A. Yeverechyahu, Ann. of Phys. 95 (1975) 35

60) F.C. Williams Jr., Phys. Lett. 31B (1970) 184

61) A.M. Bernstein, in Advances in nuclear physics, vol. 3, ed. M. Baranger and E. Vogt (Plenum, New York, 1969) p. 325

62) L.R. Elton, Nuclear radii, Landolt-Börnstein, New Series vol. I/4 (Springer, Berlin, 1967) p. 1

63) J. Jänecke, Nucl. Phys. A181 (1972) 49

64) I. Angeli and M. Csatlos, Nucl. Phys. A288 (1977) 480;

I. Angeli, M. Beiner, R.J. Lombard and D. Mas, J. Phys. G6 (1980) 303

65) B.A. Brown, C.R. Bronk and P.E. Hodgson, J. Phys. G10 (1984) 1683

66) E. Wesolowski, J. Phys. G. 10 (1984) 321; ibid. 11 (1985) 909

67) M.N. Harakeh, Proc. 4th Int. Conf. on Nuclear reaction mechanisms, ed. E. Gadioli, Varenna, Italy (June 1985) p. 353

68) D. Zawischa, J. Speth and D. Pal, Nucl. Phys. A311 (1978) 445

69) Y. Abgrall, B. Morand, E. Caurier and B. Grammaticos, Nucl. Phys. A346 (1980) 431

70) S. Jang, Nucl. Phys. A401 (1983) 303

71) M. Buenerd, D. Lebrun, Ph. Martin, P. de Saintignon and G. Perrin, Phys. Rev. Lett. 45 (1980) 1667

72) U. Garg, P. Bogucki, J.D. Bronson, Y.-W. Lui, C.M. Rozsa and D.H. Youngblood, Phys. Rev. Lett. $45(1980) 1670$

73) S. Brandenburg, R. De Leo, A.G. Drentje, M.N. Harakeh, H. Janszen and A. van der Woude, Phys. Rev. Lett. 49 (1982) 1687

74) H.P. Morsch, M. Rogge, P. Turek, C. Mayer-Böricke and P. Decowski, Phys. Rev. C25 (1982) 2939

75) J. Jänecke, Phys. Lett. 103B (1981) 1

76) J. Jänecke and E. Comay, Phys. Lett. 140B (1984) 1 